\title{
Bex1 is essential for ciliogenesis and harbours biomolecular condensate-forming capacity
}

\author{
Emi Hibino ${ }^{1,2 \dagger}{ }^{\dagger}$, Yusuke Ichiyama ${ }^{3 \dagger}$, Atsushi Tsukamura ${ }^{1,4,5+}{ }^{+}$, Yosuke Senju ${ }^{6}$, Takao Morimune ${ }^{1,4,5}$, Masahito Ohji ${ }^{3}$, \\ Yoshihiro Maruo ${ }^{4}$, Masaki Nishimura ${ }^{1}$ and Masaki Mori ${ }^{1,5^{*}}$ (D)
}

\begin{abstract}
Background: Primary cilia are sensory organelles crucial for organ development. The pivotal structure of the primary cilia is a microtubule that is generated via tubulin polymerization reaction that occurs in the basal body. It remains to be elucidated how molecules with distinct physicochemical properties contribute to the formation of the primary cilia.

Results: Here we show that brain expressed X-linked 1 (Bex 1) plays an essential role in tubulin polymerization and primary cilia formation. The Bex 1 protein shows the physicochemical property of being an intrinsically disordered protein (IDP). Bex1 shows cell density-dependent accumulation as a condensate either in nucleoli at a low cell density or at the apical cell surface at a high cell density. The apical Bex1 localizes to the basal body. Bex 1 knockout mice present ciliopathy phenotypes and exhibit ciliary defects in the retina and striatum. Bex 1 recombinant protein shows binding capacity to guanosine triphosphate (GTP) and forms the condensate that facilitates tubulin polymerization in the reconstituted system.
\end{abstract}

Conclusions: Our data reveals that Bex1 plays an essential role for the primary cilia formation through providing the reaction field for the tubulin polymerization.

Keywords: Intrinsically disordered protein (IDP), Primary cilia, Bex1, Juvenility-associated genes (JAGs), Tubulin polymerization

\section{Background}

Juvenile animals possess physiological characteristics distinct from those of adults. These features include growth, maturation, regeneration, and plasticity capabilities, among others. With the aim of revealing the molecular machinery underlying juvenile-predominant capacities, we identified juvenility-associated genes (JAGs) as genes

\footnotetext{
*Correspondence: mori.masaki@ncvc.go.jp

†Emi Hibino, Yusuke Ichiyama and Atsushi Tsukamura contributed equally.

${ }^{5}$ Department of Vascular Physiology, National Cerebral

and Cardiovascular Centre Research Institute, 6-1 Kishibe-Shimmachi,

Suita, Osaka 564-8565, Japan

Full list of author information is available at the end of the article
}

that are selectively highly expressed in juvenile cells [1]. Here, we show the physiological role of Bex1, a JAG that is encoded on the $\mathrm{X}$ chromosome and predominantly expressed in juvenile organs in mice. The molecular function of Bex1 largely remains to be determined, although its roles have been reported in the contexts of tumorigenesis [2-4], muscle regeneration [5] and the response to heart failure [6].

Cells possess cilia, which are hair-like structures that modulate and sense the extracellular environment. The propeller-like motion or periodic fluttering of motile cilia affects the extracellular space by generating flows or waves of extracellular fluids, thereby determining 
right-left asymmetry [7], or driving the cerebrospinal fluid flow [8]. Nonmotile cilia, or primary cilia, are found in numerous cell types in vertebrates [9] and sense physical and chemical inputs, thus adjusting the cellular response to external environment [10-13]. The mechanosensing ability of cilia contributes to the determination of tubular diameter in vascular vessels and renal tubules [14]. Their ligand sensing ability is exemplified by a role in Hedgehog signalling [15], which regulates cell growth [16] and causes medulloblastoma when it occurs in excess [17].

The different types of cilia are formed by distinct mechanisms, as implied by the identification of gene mutations that affect ciliogenesis. Ciliopathies can be subdivided into motile and nonmotile ciliopathies based on the major clinical manifestations $[18,19]$. Defects in motile cilia are found in primary ciliary dyskinesia and manifest as situs inversus (reversal of left-right asymmetry) and chronic respiratory infections that are due to dyskinetic airway cilia. Dysfunctional primary cilia are found in primary ciliopathies including Joubert syndrome that is characterized by cerebellar hypoplasia, retinal dysplasia and renal cystic disease [20,21].

The formation of the primary cilium starts with the migration of centrioles to the cell surface. The centrioles contain nine microtubule triplets and mature into a centrosome that is referred to as a basal body in the context of ciliogenesis [22, 23]. Basal body maturation occurs along with the accumulation of components essential for tubulin nucleation to form the molecular condensed region known as the pericentriolar matrix (PCM [24];). The PCM forms the reaction field for tubulin polymerization. The molecular components of the PCM include Pericentrin [25], Cep152/Asl, Cep192/SPD-2, Cep215/ Cnn, and SPD- 5 but have yet to be completely defined $[24,26]$. Tubulin polymerization is mediated by the polymerizing reaction of $\alpha-/ \beta$-tubulin heterodimers catalysed with guanosine triphosphate (GTP) as the energy source [27]. The basal body is thought to form the reaction field where microtubule synthesis occurs by supplying reaction substrates through a mechanism that has remained elusive thus far.

Cellular compartmentalization is a mechanism that increases the efficiency of molecular reactions. An intriguing feature of intrinsically disordered proteins (IDPs) is their tendency to form subcellular compartments specialized for molecular reactions [28-30]. IDPs lack a stable secondary structure due to biassed amino acid components or repeated sequence elements in intrinsically disordered regions (IDRs). IDPs induce phase separation and form biomolecular condensates such as nucleoli [31]. Concentrating components can increase reaction kinetics, thus enhancing reaction efficiency [31, 32].

In this paper, we describe a role of Bex1 as an IDP that is essential for ciliogenesis both in vitro and in vivo. Bex1 forms a condensate at the basal body of the primary cilium. Bex1 mutation causes ciliopathy phenotypes in mice. A recombinant Bex1 protein reconstitutes the Bex1 biomolecular condensates that induce the polymerization of tubulin, the pivotal structure of the primary cilium. Our findings elucidate the essential role of Bex1 biomolecular condensates in ciliogenesis.

\section{Results}

\section{Bex 1 is a JAG essential for cell growth}

To search for genes that are relevant to juvenile growth and tissue maturation, we performed transcriptome analysis and identified the Bex family of genes, whose members are expressed predominantly in juveniles rather than in adults (Fig. 1a and Additional file 1: Fig. S1a). The Bex family consists of 11 genes (Bex1, Bex2, Bex3, Bex4, Tceal1, Tceal3, Tceal5, Tceal6, Tceal7, Tceal8 and Tceal9), many of which showed ubiquitous juvenile-predominant expression in the investigated cerebral cortex tissues, cardiomyocytes and hepatocytes (Fig. 1a and Additional file 1: Fig. S1a). To study their functions in growth, we performed a cellular analysis focusing on Bex1, which showed the most restricted expression in juveniles (Additional file 1: Fig. S1a). The depletion of BEX1 in ARPE19 human retinal pigment epithelial cells (Fig. 1b) resulted in significant impairment of cellular growth (Fig. 1c,d), suggesting a role of $B E X 1$ in cell growth. To address the cell type and species dependence of this effect, we performed Bex1 depletion in Neuro2a mouse neural progenitor cells (Additional file 1: Fig. S1b) and confirmed the significant suppression of cellular growth (Additional file 1: Fig. S1c,d). These results indicated the indispensable role of Bex 1 in cell growth and led us to investigate the molecular function of Bex1.

\section{Bex1 has the physical characteristics of an IDP}

To investigate the molecular function of Bex1, we performed domain searches in protein databases such as pfam, InterPro, SMART and PROSITE. None of the analyses predicted any functional domains in the Bex1 protein. We then analysed physical properties of Bex1 protein. Structural modelling with Phyre2 [33] showed that Bex1 contained an IDR that occupied $54.0 \%$ of its entire length (Additional file 1: Fig. S2a). Long IDRs (> $40 \%$ of the entire length) were found in all the Bex family proteins, suggesting that Bex family proteins function as IDPs (Additional file 1: Fig. S2a). Further predictive analyses conducted according to the physical characteristics of Bex family proteins with the Protein DisOrder 
a Heatmap analysis of Bex family genes
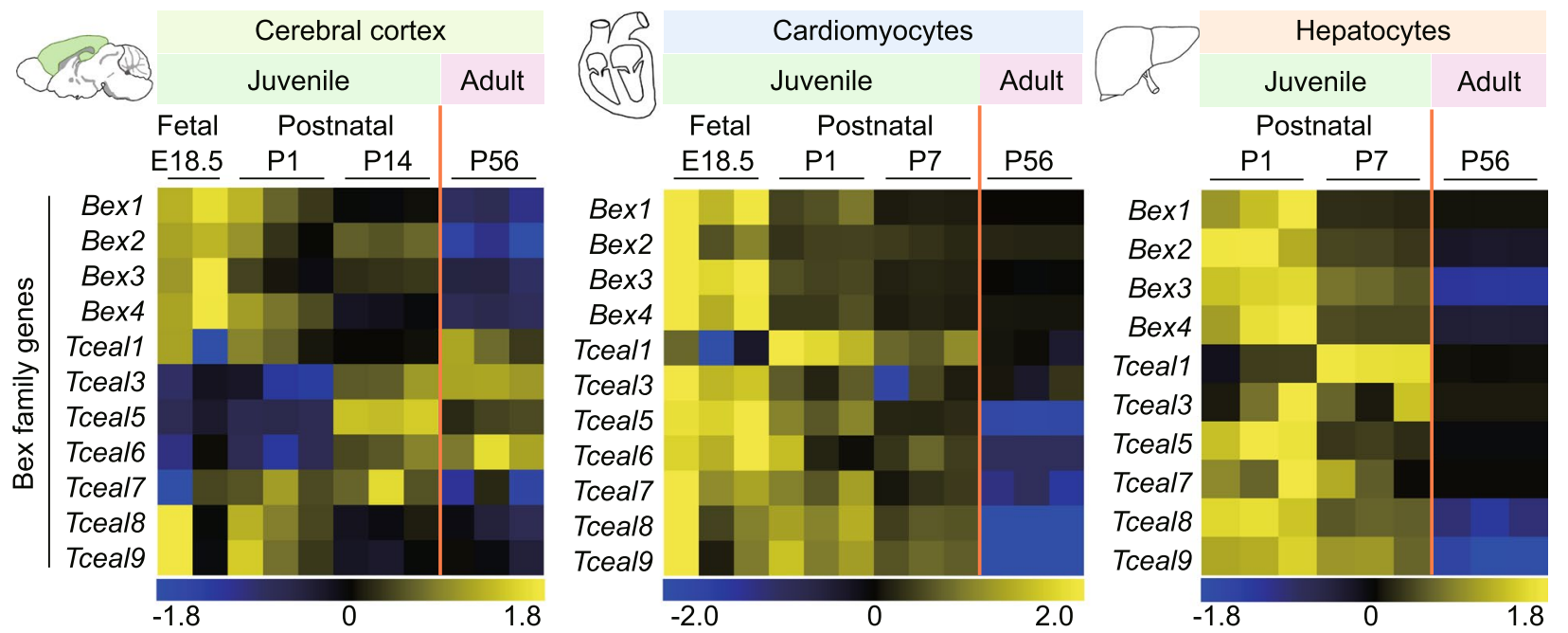

b BEX1 expression in human ARPE19 cells

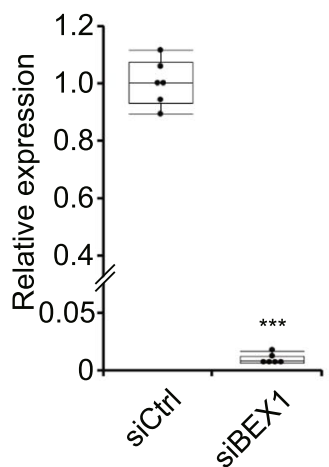

C Growth analysis with ARPE19 cells

d Number of cells
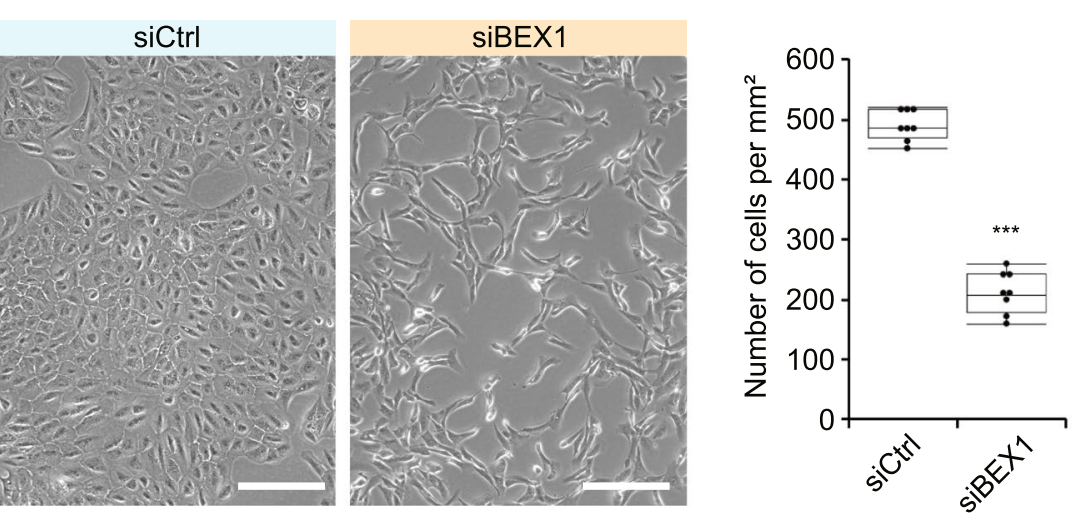

Fig. 1 Bex 1 is expressed in juvenile and is essential for cell growth. a Heatmap analysis of Bex family genes in the cerebral cortex, cardiomyocytes and hepatocytes at different embryonic (E18.5) and postnatal days (P1, P7 and P56). E, embryonic day. P, postnatal day. b qPCR analysis of BEX1 in human ARPE19 cells $48 \mathrm{~h}$ after transfection of control siRNA or BEX1 siRNA. Data were normalized to TUBB. $\mathbf{c}$ Phase contrast images of ARPE19 cells $72 \mathrm{~h}$ after transfection of control siRNA or BEX1 siRNA. Scale bar $=250 \mu \mathrm{m}$. d Number of cells per field $72 \mathrm{~h}$ after transfection with control siRNA or BEX1 siRNA. $* * * p<0.001$; Student's $t$ test. The data are presented as the means \pm standard deviations

prediction System (PrDOS) and DISOPRED3 algorithms verified that they contained highly disordered domains predominantly located in the N-terminal half of the proteins (Additional file 1: Fig. S2b).

To investigate the physical properties of the Bex1 protein via physicochemical analyses, we established a purification workflow for a recombinant Bex1 protein without any denaturing step (Fig. 2a,b). A previous attempt to analyse the Bex1 protein included the denaturing steps of boiling and the application of acidic conditions ( $\mathrm{pH}$ 5.0), which hampered the assessment of the protein under physiological conditions [34]. The

(See figure on next page.)

Fig. 2 Bex1 has the physical identity as an IDP. a The purification scheme to obtain the recombinant Bex1 protein. b Coomassie brilliant blue (CBB) staining of purified Bex1 protein. $\mathbf{c}$ Far-UV circular dichroism (CD) spectrum of the recombinant Bex 1 protein at 10, 25 and $37^{\circ} \mathrm{C}$. A typical random coil CD spectrum was detected for the Bex1 protein. The measurements of a typical structured protein, albumin, are shown for comparison. $\Theta$, molar ellipticity. c Two-dimensional nuclear magnetic resonance (2D-NMR) spectrum of the ${ }^{15} \mathrm{~N}$-labelled recombinant Bex 1 protein. The ${ }^{1} \mathrm{H}-{ }^{15} \mathrm{~N}$ heteronuclear single quantum coherence (HSQC) spectrum was measured at $\mathrm{pH} 7.3$ and 10,25 and $37^{\circ} \mathrm{C}$. The spectrum of a typical structured protein, ubiquitin, are shown for comparison. Signals observed around 6.8-7.6 ppm in Bex1 plots were derived from side-chain amide groups 
a Purification of recombinant Bex1 protein

His-Bex1 expressed in E. coli

$$
\downarrow
$$

Sonication, centrifugation

$\downarrow$

Supernatant

$\downarrow$

Ni-NTA resin

$\downarrow$

Elution

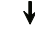

Dialysis

$\downarrow$

Purified His-Bex1 b CBB staining of recombinant Bex1 protein

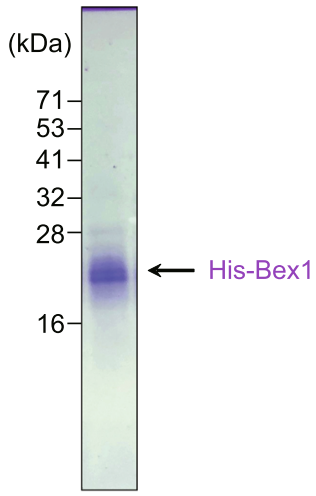

C CD spectroscopy

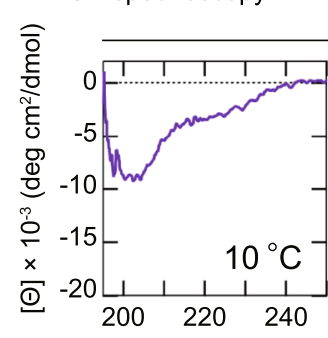

Bex1 protein
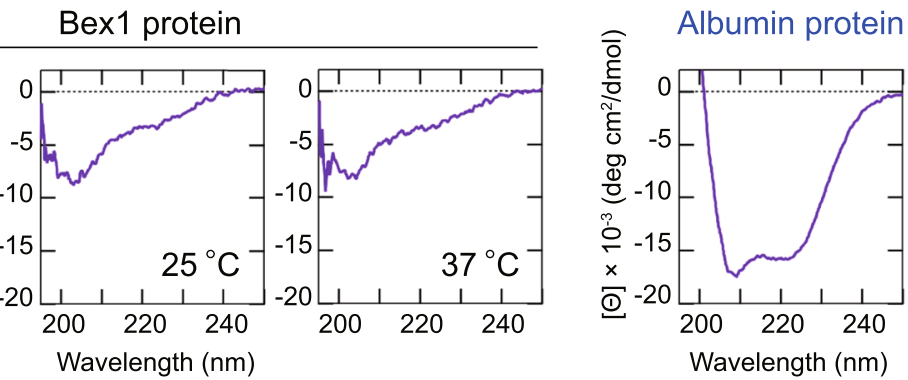

d 2D-NMR spectroscopy

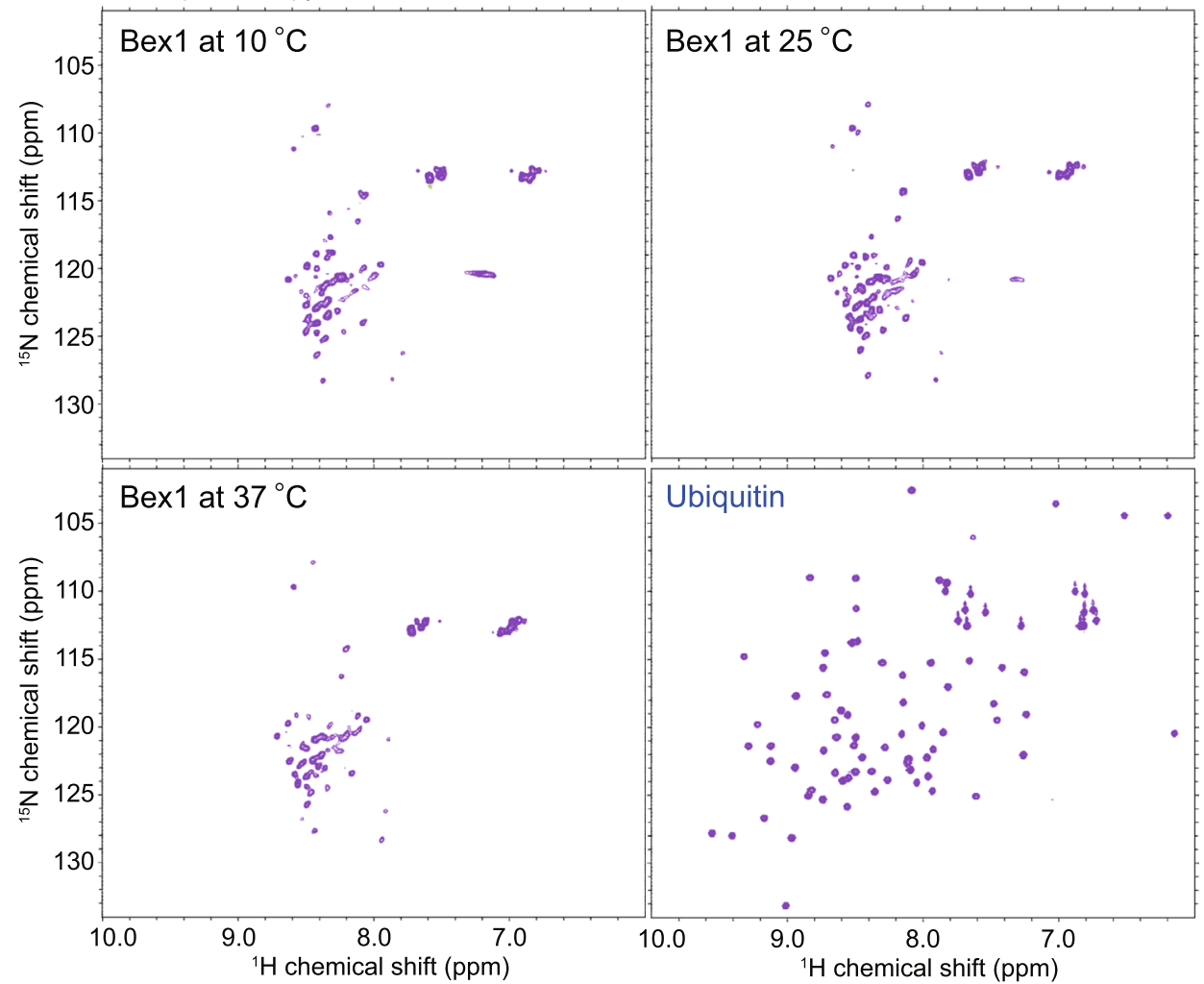

Fig. 2 (See legend on previous page.) 
native-state Bex1 recombinant protein was assessed under physiological conditions at $37^{\circ} \mathrm{C}$. Circular dichroism (CD) spectroscopy with Bex1 revealed a striking pattern that differed from that of albumin, which was investigated as a control structured protein (Fig. 2c). The CD spectrums of Bex1 was similar to an IDR of SP1 that is a well-characterized IDP [35], in that they showed a negative peak at around $200 \mathrm{~nm}$ (Additional file 1: Fig. S2c). The results suggested that the Bex1 protein lacked a stable secondary structure such as $\alpha$-helixes or $\beta$-strands (Fig. 2c). As protein structure is known to be affected by temperature, $\mathrm{CD}$ spectral analyses were conducted at temperatures of 10,25 and $37^{\circ} \mathrm{C}$ that gave similar results, confirming that the Bex1 protein did not have a stable secondary structure (Fig. 2c).

To further investigate the physical properties of the Bex1 protein, two-dimensional nuclear magnetic resonance (2D-NMR) spectroscopy was performed to analyse the tertiary structure of Bex1 under nondenaturing conditions at the different temperature of 10, 25 and $37^{\circ} \mathrm{C}$. The Bex1 spectra showed ${ }^{1} \mathrm{H}$ chemical shifts in a narrower range $(7.8-8.5 \mathrm{ppm})$ than that observed for ubiquitin, a control structured protein $(6.5-10.0 \mathrm{ppm})$, suggesting that Bex1 lacked stable tertiary structures (Fig. 2d). The 2D-NMR spectrums of Bex1 were similar to those of an IDR of TAF4 (Additional file 1: Fig. S2d, [36]). The spectral patterns concentrated at approximately $8.0 \mathrm{ppm}$ were characteristic of a protein in a random coil state [37], suggesting that the purified Bex1 protein adopts a random coil state under physiological conditions (Fig. 2d). These analyses provided evidence that Bex1 possesses the physical identity of an IDP under physiological conditions.

\section{Cell density-dependent localization of BEX1}

The physical implication that Bex1 functions as an IDP led us to next examine the biological behaviours of Bex1. We first analysed the subcellular localization of BEX1 in ARPE19 cells. BEX1 showed cell density-dependent subcellular localization. At a low cell density in which at least one side of cells was unoccupied and did not contact other cells, BEX1 localized to nucleoli (Fig. 3a). The depletion of BEX1 by siRNA transfection erased the nucleolar signal, supporting the specificity of the nucleolar signal to BEX1 (Additional file 1: Fig. S3a). The cytoplasmic signals were not suppressed by $B E X 1$ knockdown and thus considered to be nonspecific (Additional file 1: Fig. S3a). At a high cell density in which cells contacted other cells on every side, BEX1 translocated to the apical surface of cells, where Bex1 was associated with tubulin fibres (Fig. 3a,b). On the apical side of the cells, Bex1 also showed colocalization with primary cilia (Fig. 3a, "High density", insets), as shown in subsequent experiments (Fig. 4). At a high cell density, ARPE19 cells form a columnar epithelial cell shape and develop apicobasal polarity. The columnar epithelial cells develop a dense microtubule network on the apical side of the cells, where BEX1 is localized (Fig. 3b). The knockdown of BEX1 led to defective formation of the apical microtubule network and flattening of cell layers (Fig. $3 \mathrm{~b}$ ). Moreover, cells depleted of BEX1 exhibited stratification, indicating that the cells lost their epithelial properties (Fig. 3c). The loss of epithelial properties was further confirmed by examining cell-cell junction proteins, and it was found that intercellular junctions formed by $\alpha$-catenin and $\beta$-catenin were lost in BEX1-depleted cells (Fig. 3d). Cell density-dependent localization was also examined in Madin-Darby canine kidney (MDCK) cells, which form a well-polarized epithelial structure. Consistent with the findings in ARPE19 cells, Bex1 localized to nucleoli at a low cell density and to the apical microtubule network at a high cell density (Fig. 3e). Bex1 localized to the apical side of cells and showed colocalization with the microtubule network (Fig. 3f). These findings suggest a role of Bex1 in the formation of the apical microtubule network and the maintenance of apicobasal polarity.

We further investigated the biological relevance of the nucleolar localization of BEX1 at a low cell density. Nucleoli show an internal structure (Additional file 1: Fig. S3b, [38]) that can be delineated by staining with

\footnotetext{
(See figure on next page.)

Fig. 3 Bex1 localizes to nucleoli and apical side of cells in the cell density-dependent manner. a Immunostaining of BEX at different cell densities in ARPE19 cells. At a low cell density, BEX1 localized to nucleoli, which were stained with a fibrillarin (FBL) antibody. At a high cell density, BEX1 showed a condensed pattern that colocalized with a-tubulin. The inset shows the localization of the BEX1 condensate (arrowhead) to tubulin fibres. Nuclei were stained with DAPI. Scale bar $=25 \mu \mathrm{m}$. b Reconstructed z-axis view of ARPE19 cells at the high cell density. In control knockdown cells (siCtrl), the immunostaining showed localization of BEX1 to the apical side of cells where tubulin was enriched (arrowhead). In BEX1-depleted cells (siBEX1), the enrichment of tubulin at the apical surface was lost and cells were flattened. c Reconstructed z-axis view of ARPE19 cells showing the stratification provoked by BEX1 knockdown. Arrowheads indicate the cells overriding other cells. Scale bar $=25 \mu \mathrm{m}$. $\mathbf{d}$ Cell scattering was induced by BEX1 knockdown. Immunostaining of cell-cell junction protein a-catenin and $\beta$-catenin and cytoskeletal protein Vimentin and Filamin. ARPE19 cells were transfected with control siRNA or BEX1 siRNA $96 \mathrm{~h}$ prior to the staining. Nuclei were counterstained with DAPI. Scale bar $=25 \mu \mathrm{m}$. e Immunostaining of Bex 1 at different cell densities in MDCK cells. At a low cell density, Bex 1 showed localization to nucleoli which were stained with FBL antibody. At a high cell density, Bex1 showed a condensed pattern which colocalized to a-tubulin. Nuclei were stained with DAPI. Scale bar $=25 \mu \mathrm{m}$. $\mathbf{f}$ The $X Y$ view and the corresponding reconstructed $z$-axis view of MDCK cells at the high cell density. The immunostaining showed localization of BEX1 to the apical side of cells where tubulin was enriched. Scale bar $=25 \mu \mathrm{m}$
} 
a Cell density-dependent localization of BEX1 in ARPE19 cells

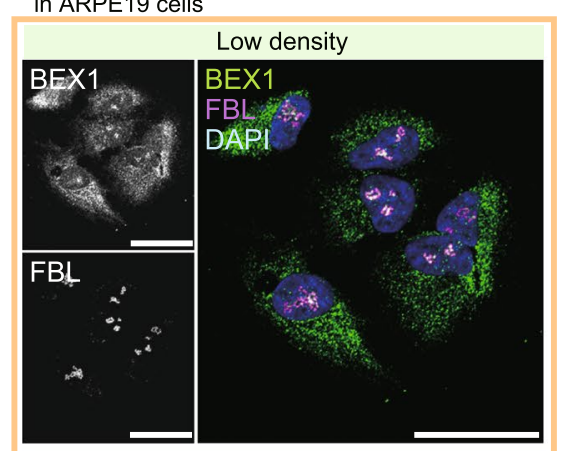

High density

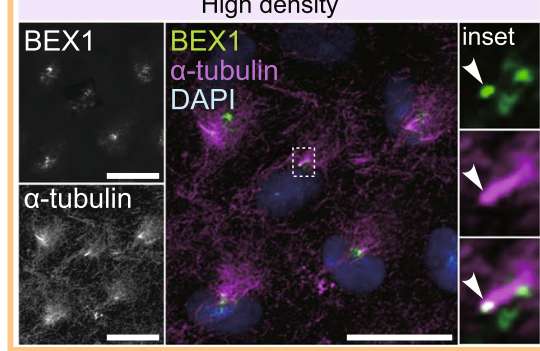

d Cell scattering induced by BEX1 loss

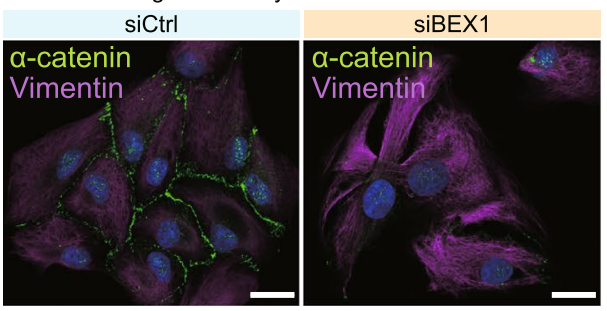

e Cell density-dependent localization of Bex1 in MDCK cells

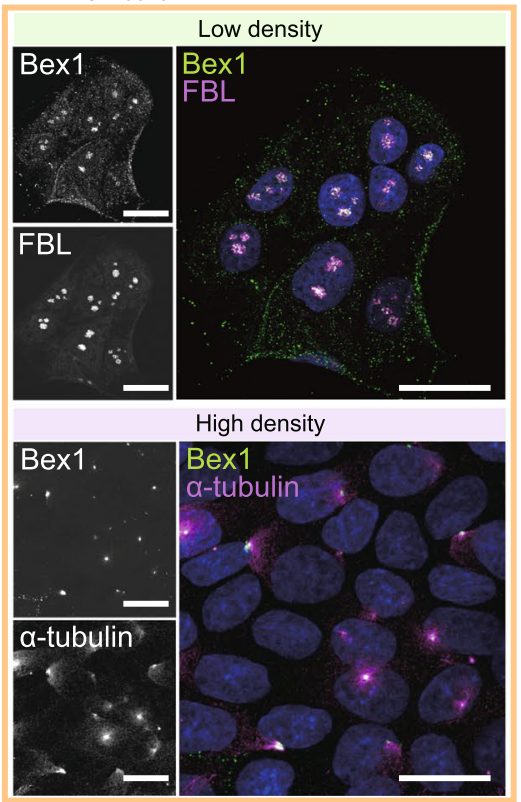

b BEX1 depletion leads to loss of apical microtubules
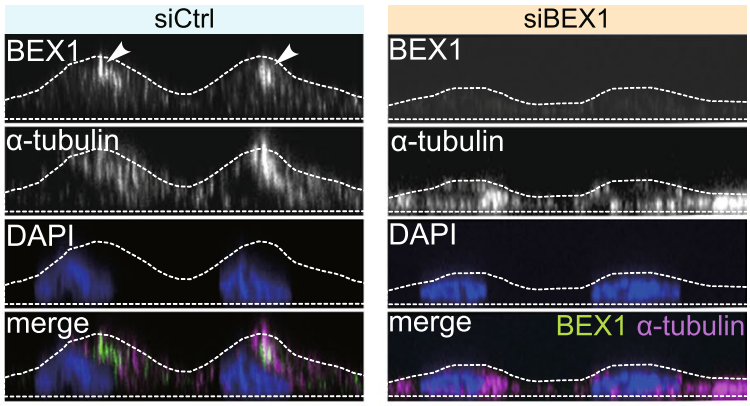

C BEX1 loss provokes stratification
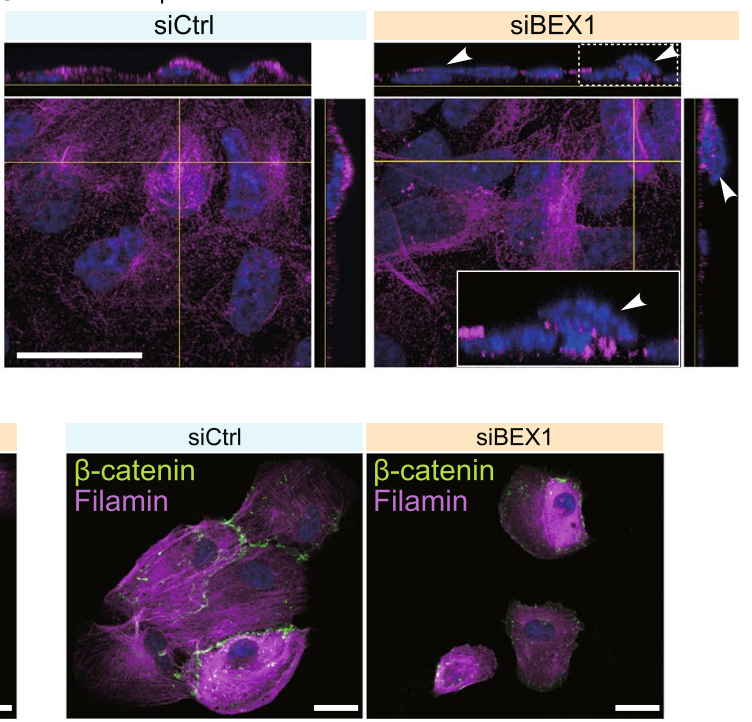

f Apical localization of Bex1 in MDCK cells

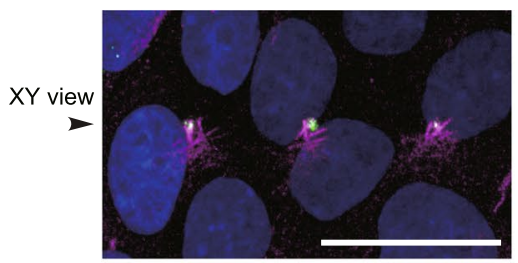

Z view

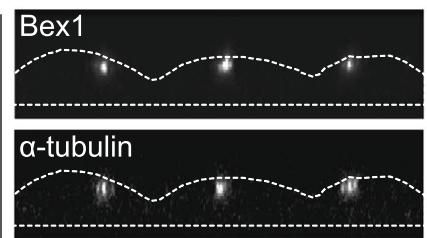

DAPI

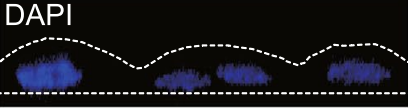

merge

BEX1 a-tubulin

Fig. 3 (See legend on previous page.) 
fibrillarin (FBL), which localizes to the dense fibrillar component (DFC [39];), and Nucleolin, which localizes to the granular component (GC [40];). BEX1 immunostaining revealed clearer overlap with FBL than with Nucleolin, suggesting that BEX1 localizes to the DFC (Additional file 1: Fig. S3). We next investigated the potential role of BEX1 in the DFC of the nucleoli. The depletion of BEX1 caused perturbation of the FBL staining pattern, suggesting the occurrence of DFC deformation (Additional file 1: Fig. S3d). The sphericity of the FBL staining patterns was significantly reduced by $B E X 1$ depletion, suggesting that $B E X 1$ plays a role in maintaining the morphology of the DFC (Additional file 1: Fig. S3e,f).

\section{BEX1 localizes to the ciliary basal body and is indispensable for ciliogenesis}

We further analysed the apical localization of BEX1 in ARPE19 cells. ARPE19 cells develop primary cilia at a high cell density. We found that BEX1 localized to the base of the primary cilia (Fig. 4a). Further analysis according to the components of the primary cilia revealed that BEX1 colocalized with PCNT, a component of the pericentriolar matrix (Fig. 4b). BEX1 knockdown abrogated the formation of the primary cilia, demonstrating the indispensable role of BEX1 in ciliogenesis (Fig. 4c,d and Additional file 1: S4a-c). We also investigate whether the cilia disruption was caused by loss of cell polarity. TGF- $\beta$ treatment induces epithelial mesenchymal transition of ARPE19 cells and provokes loss of apicobasal polarity [41-43]. The cilia formation was not appreciably affected by the loss of apicobasal polarity in ARPE19 cells, indicating the ciliary loss observed in BEX1-depleted cells was not due to the loss of cell polarity (Additional file 1 : Fig. S4d).

The primary cilium is not restricted to polarized epithelial cells and is also found in NIH3T3 cells, a type of mesenchymal cells (Fig. 4e). Bex1 knockdown in NIH3T3 cells (Fig. 4f) compromised the formation of primary cilia (Fig. 4e). We noted that a small number of cells $(9.1 \%)$ exhibited supernumerary cilia (Fig. 4g,h). The molecular aetiology of supernumerary cilia was subsequently investigated (Fig. 5). If cilia were detected at all, the length of the primary cilia was significantly shortened, indicating that ciliogenesis was compromised by Bex1 depletion (Fig. 4i,j). Overexpression of BEX1 did not affect ciliogenesis and PCNT localization (Additional file 1: Fig. $\mathrm{S} 4 \mathrm{e}, \mathrm{f})$. Thus, BEX1 protein localization at the base of the primary cilium is indispensable for proper ciliogenesis.

\section{BEX1 depletion from the midbody provokes cytokinesis failure, underlying the formation of supernumerary cilia} The colocalization of BEX1 with apical microtubules and primary cilia led us to investigate the potential localization of BEX1 to the other tubulin-enriched subcellular apparatus. BEX1 immunostaining revealed the accumulation of BEX1 in the midbody, where tubulin forms dense microtubules in dividing ARPE19 cells (Additional file 1: Fig. S5a). BEX1 knockdown hampered the formation of midbodies with typical tubulin accumulation (Additional file 1: Fig. S5b), resulting in the elongation of the cells as a result of compromised abscission in ARPE19 cells (Additional file 1: Fig. S5c).

Additionally, in NIH3T3 cells, we found that Bex1 localized to the midbody (Additional file 1: Fig. S5d). Bex1 depletion led to abnormal nuclei with increased foci of PCNT, an essential component of the centrosome, suggesting that the failure of cell division led to the supernumerary centrosomes (Additional file 1: Fig. S5e,f). BEX1 depletion caused the increased PCNT foci also in ARPE19 cells (Additional file 1: Fig. S5g). The centrosome functions as the basal body in the context of ciliogenesis. We actually observed that the increased number of centrosomes contributed to the formation of supernumerary cilia in Bex1-depleted NIH3T3 cells (Additional file 1: Fig. S5h,i).

Compared to the results obtained in ARPE19 cells, in which BEX1 depletion abrogated ciliogenesis nearly completely, NIH3T3 cells exhibited remaining cilia with aberrant lengths and numbers (Fig. 4g-j), implying a compensatory mechanism for Bex1-dependent

(See figure on next page.)

Fig. 4 Bex 1 localizes to the ciliary base and promotes ciliogenesis. a Immunofluorescence analysis of BEX1 and primary cilia in human ARPE19 cells. The primary cilia were stained with the ARL13B antibody. Bex1 localized to the base of primary cilia. Nuclei were stained with DAPI. Scale bar $=10$ $\mu \mathrm{m}$. $\mathbf{b}$ Immunofluorescence analysis of BEX1, PCNT and ARL13B in human ARPE19 cells. BEX1 and PCNT colocalize at the ciliary base. Scale bar $=5$ $\mu \mathrm{m}$. $\mathbf{c}$ Immunofluorescence analysis for the primary cilia in ARPE19 cells transfected with control siRNA or BEX1 siRNA. Cilia formation was abrogated by the BEX1 depletion. Nuclei were stained with DAPI. Scale bar $=25 \mu \mathrm{m}$. d Frequency of ciliated cells after transfection with control siRNA or BEX1 siRNA. The number of ciliated cells was counted in the experiment (c). e Immunofluorescence analysis for the primary cilia in mouse NIH3T3 cells transfected with control siRNA or Bex 1 siRNA. Primary cilia were visualized with the acetylated tubulin (Ac-tubulin) antibody. Nuclei were stained with DAPI. Scale bar $=25 \mu \mathrm{m}$. $\mathbf{f}$ qPCR analysis for the expression of Bex1 in NIH3T3 cells transfected with control siRNA or Bex1 siRNA. Data were normalized to Tubb5. g Supernumerary cilia observed in Bex1-depleted cells. The primary cilia were stained with antibodies against Ac-tubulin and IFT88. Nuclei were stained with DAPI. Scale bar $=10 \mu \mathrm{m}$. $\mathbf{h}$ Distribution of the number of primary cilia in a cell after transfection with control siRNA or Bex 1 siRNA. A total of 9.1\% of Bex 1-depleted cells exhibited supernumerary cilia (denoted as " 2 or more"). i Length ( $\mu$ m) distribution of the cilia in NIH3T3 cells transfected with control siRNA or Bex 1 siRNA. $\mathbf{j}$ Average length $(\mu \mathrm{m})$ of the primary cilia in NIH3T3 cells after transfection of control siRNA or Bex1 siRNA. *** $p<0.001$; Student's $t$ test. The data are presented as the means \pm standard deviations 
a Immunofluorescence analysis of BEX1 and primary cilia in ARPE19 cells

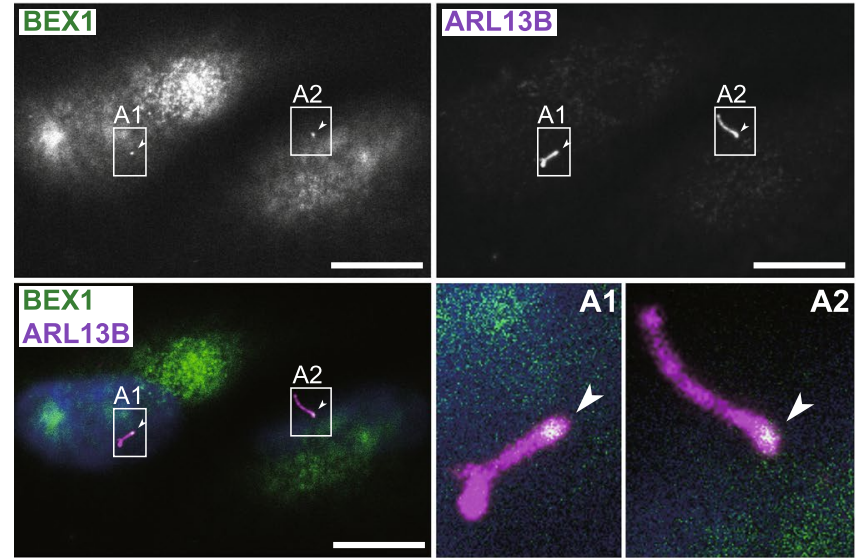

C Immunofluorescence analysis of primary cilia

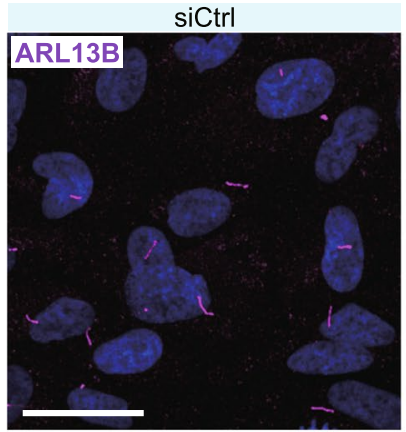

b

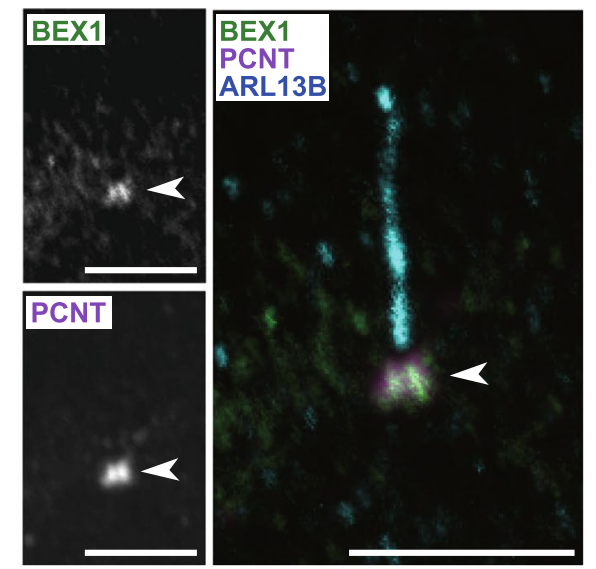

d Frequency of ciliated cells
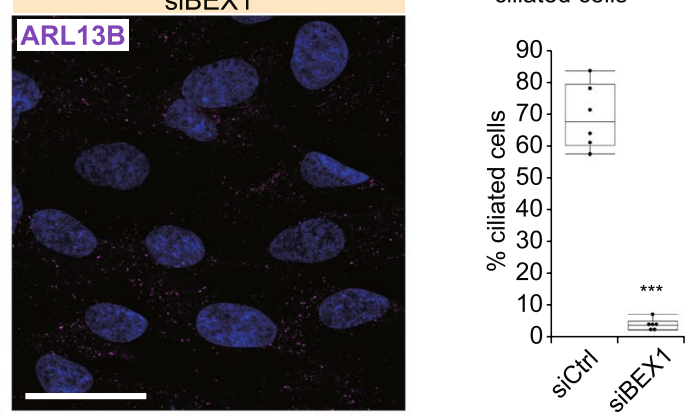

e Immunofluorescence analysis of primary cilia in $\mathrm{NIH} 3 \mathrm{~T} 3$ cells $\mathbf{f}$ Bex1 expression siCtrl siBex1

g Supernumerary cilia in Bex1-depleted cells

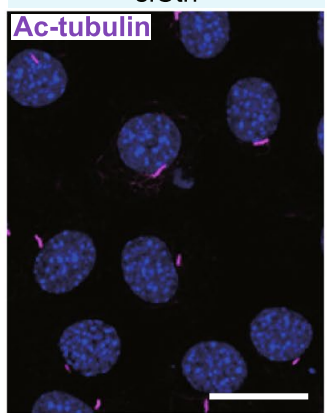

h Distribution of ciliary numbers

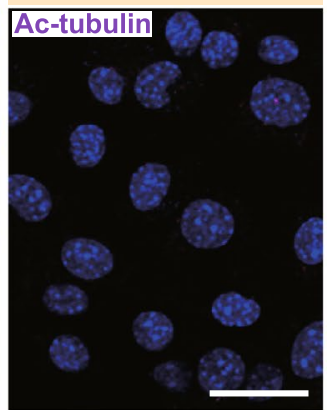

i in $\mathrm{NIH} 3 \mathrm{~T} 3$ cells
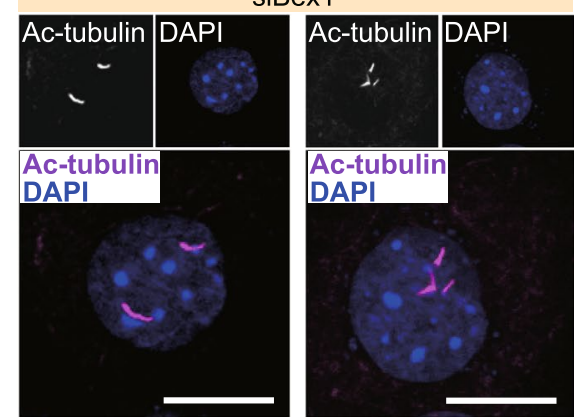

j Average length

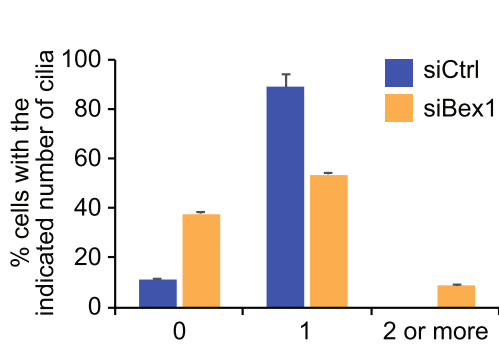

Number of cilia
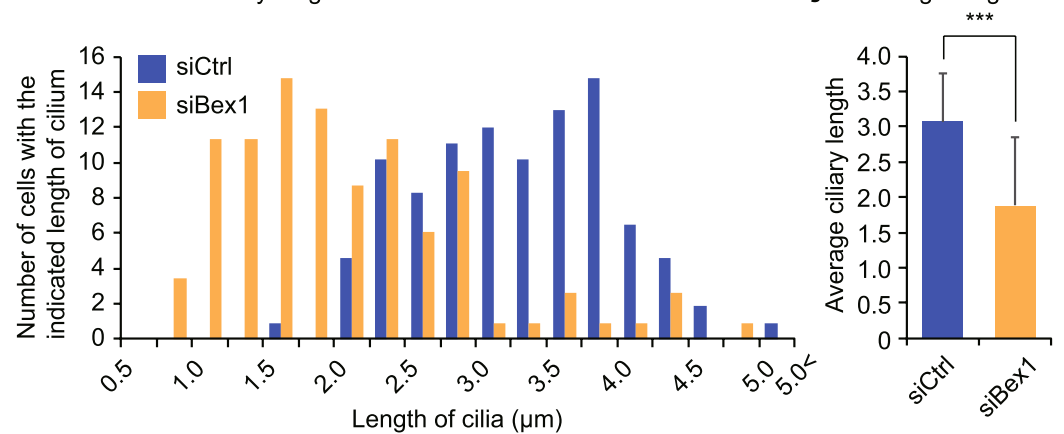

Fig. 4 (See legend on previous page.) 
ciliogenesis. This finding prompted us to investigate the potential mechanism that compensated for cilium formation in the absence of Bex1 expression in NIH3T3 cells. Bex2 shared the highest structural similarity with Bex1 among the members of the Bex family and was expressed in NIH3T3 cells (Additional file 1: Fig. S6a). BEX2 expression was low in ARPE19 cells (Additional file 1: Fig. S6b). Although Bex2 depletion showed minor effects on ciliogenesis (Additional file 1: Fig. S6), the simultaneous depletion of Bex1 and Bex2 resulted in the complete suppression of supernumerary cilia, suggesting that Bex 2 plays a compensatory role in ciliogenesis in the absence of Bex1 (Additional file 1: Fig. S6c,d). Thus, the increase in centrosomes due to Bex1 depletion underlies the formation of supernumerary cilia, which is mediated by the compensatory role of Bex 2 .

\section{Bex1 mutant mice exhibit ciliopathy phenotypes}

We next sought to determine a role of Bex1 in vivo. We established Bex 1 mutant mice via CRISPR-Cas9 genome editing [44-46], Additional file 1: Fig. S7a). We utilized a strain with a frameshift mutation in Bex1 (Additional file 1: Fig. S7b) in which we confirmed that no mutation occurred in Bex2 (Additional file 1: Fig. S7c). The loss of the Bex1 protein was confirmed using a newly developed antibody that is responsive to mouse Bex1 (Fig. 5a). Bex1null male $\mathrm{e}^{-/ \mathrm{Y}}$ and female $\mathrm{e}^{-/-}$mice were viable and fertile.

Female mutant mice showed lateral or bilateral ocular turbidity more frequently than male mice (Fig. $5 \mathrm{~b}$ and Additional file 1: Fig. S7d), indicating that Bex1 shows sex-dependent biological relevance. The female mutants also showed a more severe phenotype causing maldevelopment of the eyeball manifesting as anophthalmia (the absence of an eye, Fig. 5c,d). Histological analyses of the eyeballs of WT and Bex1 mutant mice revealed thickening of the corneal epithelium (Fig. 5e,f) and disoriented formation of retinal epithelial layers (Fig. $5 \mathrm{~g}$ ), in line with the in vitro findings in ARPE19 cells, in which BEX1 knockdown led to a loss of apicobasal polarity and stratification of cells (Fig. 3c).

For the determination of the phenotypes, we focused on female homozygous mutant mice. The Bex1 mutant mice also presented abdominal swelling that was caused by enlarged kidneys with multiple cysts (Fig. 5h). Renal histological analysis revealed large cystic lesions (Fig. 5i) and dilatation of the renal tubules, accompanied by glomerular involution, confirming that the mice were affected by polycystic kidney disease (Fig. 5j). Moreover, Bex1 mutant mice exhibited an increased head circumference due to hydrocephalus accompanied by hypoplasia of the cerebellar vermis (Fig. 5k). The cerebellar vermis was primarily affected, although secondary hydrocephalus caused deformation of the entire cerebellum. The histological analysis of the cerebellum revealed thinning of the cerebellar granule cell layers (Fig. 5l). The motile cilia of the airway epithelium were not affected by Bex 1 mutation, confirming the physiological relevance of Bex1 in primary cilia, not motile cilia (Additional file 1: Fig. S7e). Appreciable defects were not found in other tissues such as skin, heart, liver and gut. We did not observe worsening of phenotypes in aged animals. This constellation of symptoms (ocular dysgenesis, polycystic kidney disease and cerebellar hypoplasia) was in accord with ciliopathy phenotypes and suggested that the defects were due to ciliary dysfunction [47-49].

We next examined the influence of Bexl mutation on the formation of primary cilia in Bex 1 mutant mice. Here, we investigated the retinal pigment epithelium (RPE) of the eyeball (Fig. 6a) and the striatum of the brain (Fig. 6d) as tissues rich in primary cilia. The primary cilia of RPE cells are indispensable for the proper maturation and functions of the cells [50-52]. Immunostaining of cilia with an ARL13B antibody revealed significantly fewer primary cilia in the retinal pigment epithelial cells of Bex1 mutant mice than in those of WT mice (Fig. 6b,c). Consistent with the findings in vitro,

\footnotetext{
(See figure on next page.)

Fig. 5 Bex 1 mutant mice exhibit ciliopathy phenotypes. a Western blot analysis for Bex 1 in a Bex 1 knockout and control wild type (WT) mice. The brain tissue lysates were analysed for Bex 1 protein expression. Gapdh was used as loading control. b Ocular opacity caused by corneal degeneration in a Bex 1 mutant mouse. c Anophthalmia exhibited by a Bex1 mutant mouse. d Maldevelopment of the eyeball caused by the Bex 1 mutation. The maldeveloped eyeball (right) was embedded in the connective tissue. An eyeball of a WT littermate is shown for comparison (left). e Ocular histological analysis of WT and Bex 1 mutant mice. Haematoxylin and eosin (HE) staining of the eyeballs is shown. Scale bar $=500 \mu \mathrm{m}$. $\mathbf{f H E}$ histological analysis of cornea showing abnormal stratification of corneal epithelium in Bex 1 mutant mice. Scale bar $=100 \mu \mathrm{m}$. $\mathbf{g}$ Retinal histological analysis showing the disorganized formation of retinal layers and malformation of retinal pigment epithelium (RPE) in Bex 1 mutant mice. Scale bar $=100 \mu \mathrm{m}$. h Polycystic kidney diseases caused by Bex 1 mutation. The upper panel shows kidneys (R and $\mathrm{L}$ ) and spleen $(*)$. Multiple cysts were observed in the kidneys of the Bex1 mutant mouse. The arrowheads indicate cysts. The lower panel shows the appearance of the dissected kidneys (R and L). Scale bar $=1 \mathrm{~cm}$. i HE staining of the WT and the Bex 1 mutant kidneys. Bilateral multiple cysts were observed. Scale bar $=4 \mathrm{~mm}$. $\mathbf{j}$ Histological analysis at higher magnification shows dilatation of renal tubules and involuted glomerulus (arrowhead) in the Bex 1 mutant mouse. Scale bar $=100 \mu \mathrm{m}$. k Dysplasia of the cerebellar vermis accompanied by hydrocephalus with fourth ventricular enlargement in the Bex 1 mutant mouse. Scale bar $=2 \mathrm{~mm}$. I Magnification of the squared areas in $(\mathbf{k})$. The brackets indicate the hypoplasia of cerebellar granule cell layers in the Bex1 mutant mouse. Scale bar $=200 \mu \mathrm{m}$
} 


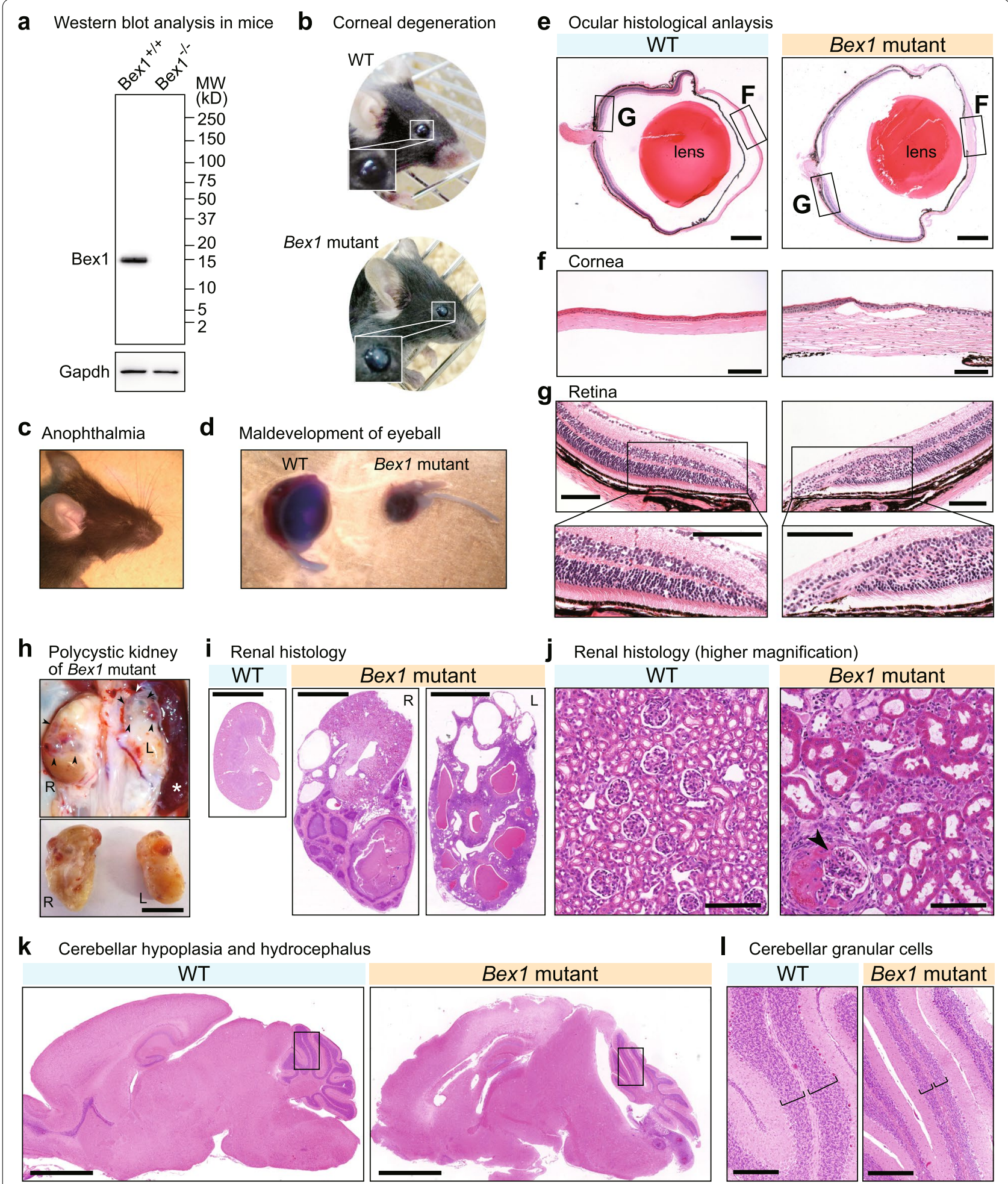

Fig. 5 (See legend on previous page.)

supernumerary cilia were observed in RPE of KO mice (Additional file 1: Fig. S7f). Additionally, in the striatum (Fig. 6d), significantly fewer primary cilia were found in
Bex1 mutant mice than in WT mice (Fig. 6e,f). These findings indicate that $B e x 1$ is indispensable for ciliogenesis in mice. 
a Analysis for primary cilia b Immunohistochemistry of primary cilia in RPE in the mouse eyeball

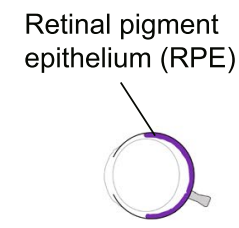

d Analysis for primary cilia in the striatum of the mouse brain
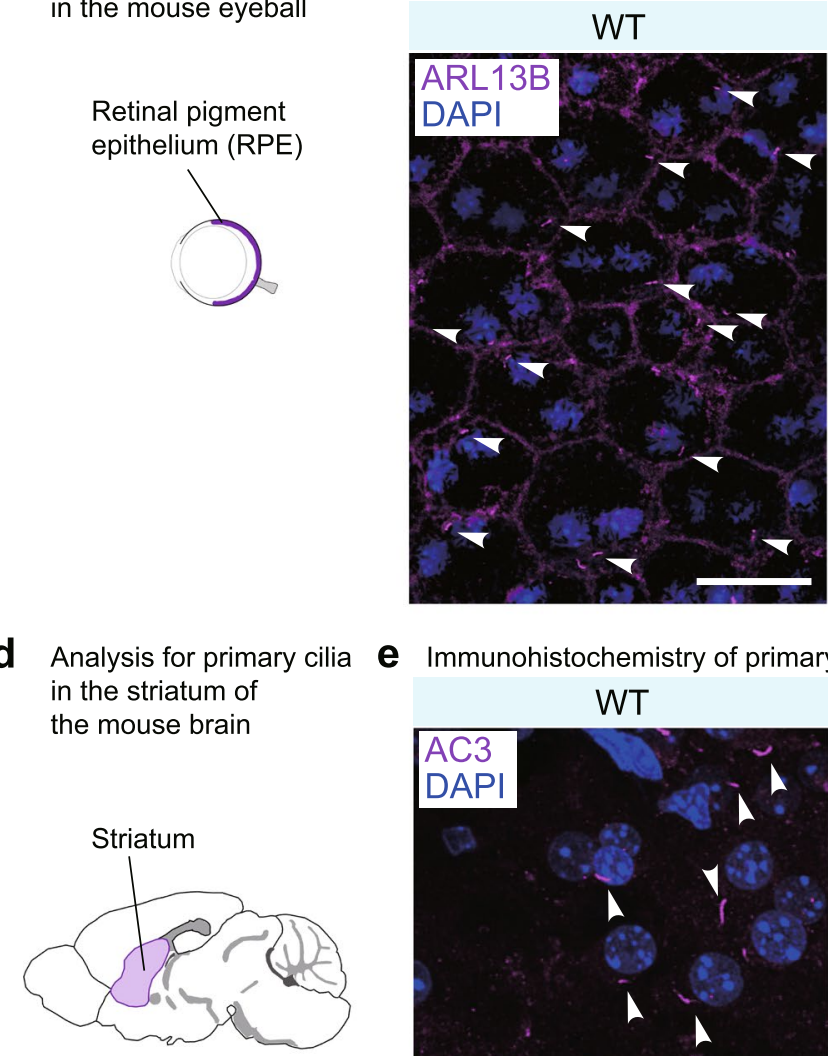

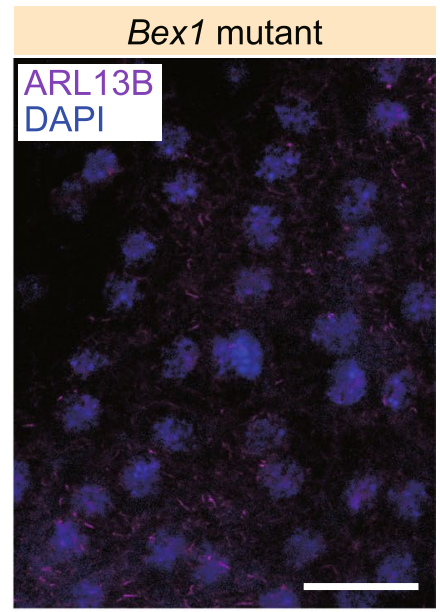

C Cells with primary cilia in RPE

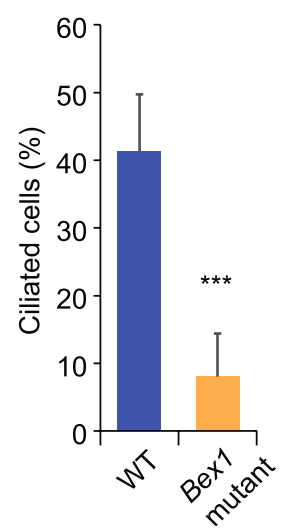

f Cells with primary cilia in striatum

WT

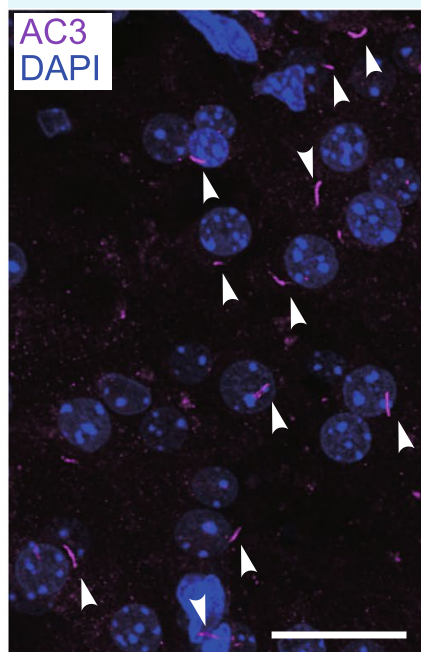

Bex1 mutant

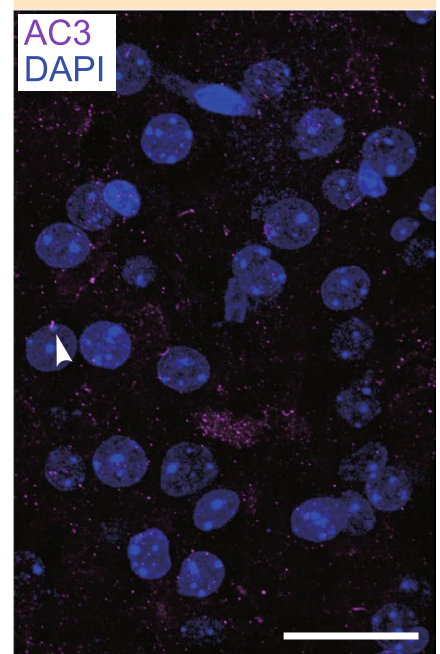

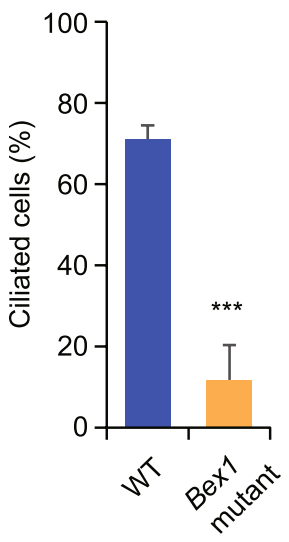

Fig. 6 Bex 1 promotes ciliogenesis in mice. a Schematic representation of the retinal pigment epithelium (RPE) in which primary cilia were analysed. b Immunohistochemistry for the primary cilia stained with ARL13B antibody in the RPE of WT and Bex 1 mutant mice at P8. Nuclei were stained with DAPI. Scale bar $=10 \mu \mathrm{m}$. c Frequency of cells with a primary cilium in the RPE of WT and Bex 1 mutant mice. $\mathbf{d}$ Schematic representation of the striatum in the mouse brain in which primary cilia were analysed. e Immunohistochemistry for the primary cilia stained with Adenylate Cyclase 3 (AC3) antibody in the striatum of WT and Bex 1 mutant mice at P13. Nuclei were counterstained with DAPI. Scale bar $=25 \mu \mathrm{m}$. f Frequency of cells with a primary cilium in the striatum of WT and Bex 1 mutant mice. $* * * p<0.001$; Student's $t$ test. The data are presented as the means \pm standard deviations

\section{The Bex1 condensate induces tubulin polymerization}

To gain insight into how Bex1, an IDP, contributes to the formation of primary cilia, we analysed the functional property of Bex1 protein. We transfected EGFP-fused Bex1 into ARPE19 cells and observed the cells under unfixed conditions. Fluorescent microscopy revealed that EGFP-Bex1 protein formed cytoplasmic granules (Fig. 7a). The Bex1 granules colocalized with tubulin, implying that the Bex1 granules concentrate tubulin (Fig. 7b). Fluorescent recovery after photo-bleaching
(FRAP) analysis with the Bex1 granules revealed rapid recovery of fluorescence suggesting the dynamic exchanges of Bex1 molecules (Additional file 1: Fig. S8a,b). Further, to investigate the potential role of Bex1 in facilitating the tubulin polymerization, we investigated the interaction of Bex1 with GTP, a crucial substrate that tubulin utilize to polymerize. To examine the binding capacity of Bex1 with GTP, we performed 2D-NMR analysis in the presence of GTP. Upon GTP addition, Bex1 underwent significant changes in its chemical 
shift, indicating that Bex1 is bound to GTP (Fig. 7c and Additional file 1: Fig. S8c). The peak shift of Bex1 was GTP concentration-dependent with the dissociation constant $\left(K_{\mathrm{d}}\right)$ equal to $244 \pm 98 \mu \mathrm{M}$ (Fig. $7 \mathrm{~d}$ ). We also tested the binding of Bex1 to another nucleotide, ATP. The 2D-NMR analysis showed that the addition of ATP changed the spectrum of ${ }^{15} \mathrm{~N}$-labelled Bex1, indicating that Bex1 also interacted with ATP. However, unlike GTP, ATP did not show a concentration-dependent peak shift, rather, the addition of 1 and 2 equivalents of ATP decreased the peak intensity of Bex1 whereas 4 and 10 equivalents increased, indicating the chemical exchange between Bex1 and ATP was slower than that between Bex1 and GTP (Additional file 1: Fig. S8d).

We utilized an assay system in which extracted tubulin underwent polymerization under reconstituted experimental conditions. The extracted tubulin did not undergo polymerization without an appropriate supply of tubulin and GTP. The purification method that we established enabled us to investigate the role of the recombinant Bex1 protein in its native state. The recombinant Bex1 protein was purified and covalently labelled with DyLight fluorescent dye. After dialysis to remove unreacted DyLight molecules, the labelled Bex1 protein was incubated in the presence or absence of polyethylene glycol (PEG4000, Fig. 7e). In the presence, but not in the absence of PEG, the Bex1 protein formed molecular condensates (Fig. 7f, g). The requirement for PEG indicated the necessity of the macromolecular crowding effect for Bex1 condensate formation. The FRAP analysis revealed recovery of the fluorescence suggesting the presence of exchanges of Bex1 protein in the condensates (Additional file 1: Fig. S8e). Next, to determine the role of the Bex1 protein in ciliogenesis, we assessed whether the presence of Bex1 condensates was sufficient for the induction of tubulin polymerization. We incubated Bex1 condensates with tubulin protein fluorescently labelled with Alexa 488
(Fig. 7h). In the presence of Bex1 condensates, the tubulin protein underwent a polymerization reaction, indicating that the Bex1 condensate provided the appropriate the microenvironment for tubulin polymerization (Fig. 7i, j). In the absence of the Bex1 condensate, tubulin monomers did not undergo polymerization (Fig. 7k). Thus, Bex1 forms molecular condensates when a molecular crowding effect exists. The Bex1 condensates were sufficient to provoke tubulin polymerization under reconstituted conditions. The tubulin polymerization is pivotal machinery for the formation of the primary cilia, thus linking the physiological role of Bex1 to the ciliogenesis.

We characterized Bex1 as a juvenile-expressed IDP that showed cell density-dependent localization to nucleoli and the apical side of cells, where Bex1 localizes to the basal body (Fig. 7l). Bex1 promotes the formation of primary cilia in both cells and mice, and Bex1 mutations cause ciliopathy phenotypes in mice. The Bex1 protein is capable of forming condensates that facilitate tubulin polymerization.

\section{Discussion}

In this paper, we have described a role of Bex1 involving cell density-dependent localization to either nucleoli or the apical side of cells. Nucleolar Bex1 was found to be located in DFCs and to be indispensable for the maintenance of DFC morphology. The colocalization of Bex1 with apical microtubules was clearly observed in confluent and polarized epithelial cells. In ARPE19 cells, the localization of BEX1 was specifically found at the base of the primary cilia. Immunostaining studies revealed the colocalization of BEX1 with PCNT, which is a fundamental component of the basal body, indicating that BEX1 functions for the formation of primary cilia.

Knockdown experiments in cultured cells demonstrated that Bex1 promotes proper ciliogenesis. Mutant mouse analyses revealed that Bex1 mutation

\footnotetext{
(See figure on next page.)

Fig. 7 Bex 1 reconstitutes tubulin polymerization. a Image derived from unfixed cells expressing EGFP-Bex1. EGFP-Bex1 forms cytoplasmic granules. Scale bar $=5 \mu \mathrm{m}$. b Immunostaining for a-tubulin in the cells expressing EGFP-Bex1. The EGFP-Bex1 granules accumulate a-tubulin. Scale bar $=5 \mu \mathrm{m}$. c The 2D-NMR spectroscopy with ${ }^{15} \mathrm{~N}$-labelled recombinant Bex 1 protein in the presence of indicated molar ratios of Bex 1 to guanosine triphosphate (GTP). The ${ }^{1} \mathrm{H}-{ }^{15} \mathrm{~N} \mathrm{HSQC}$ spectra were measured at $\mathrm{pH} 7.3$ and $25^{\circ} \mathrm{C}$. The increased GTP provoked the peak shifts (indicated by arrowheads) in a dose-dependent manner. $\mathbf{d}$ Calculation of the dissociation constant $\left(K_{d}\right)$ for Bex 1 and GTP. The distance of the peak shifts ( $\triangle \delta$ ) obtained in (C) was plotted as the filled dots to calculate the dissociation constant of Bex 1 and GTP. For comparison, the plots from the upper right peak that did not shift were shown as the open dots. e Concentrations of reagents for the analysis of the Bex 1 condensate formation. $\mathbf{f}$ Formation of Bex 1 condensates in the presence or absence of PEG4000. Recombinant Bex 1 protein was labelled with DyLight fluorescent dye. Scale bar $=5$ $\mu \mathrm{m} . \mathbf{g}$ The magnified view of the Bex 1 condensate. Scale bar $=1 \mu \mathrm{m}$. $\mathbf{h}$ Concentrations of reagents for the analysis of tubulin polymerization in the presence of the Bex 1 condensates. $\mathbf{i}$ Representative images of polymerized tubulin (magenta) provoked by the presence of the Bex 1 condensates (green). Tubulin protein was labelled with Alexa fluorescent dye. Scale bar $=1 \mu \mathrm{m}$. $\mathbf{j}$ Histogram of the length ( $\mu \mathrm{m}$ ) of the polymerized tubulin in the presence of the Bex 1 condensates. $\mathbf{k}$ Length $(\mu \mathrm{m})$ of the polymerized tubulin. Without recombinant Bex 1 protein, no tubulin polymerization was observed. I Schematic representation of the cell density-dependent role of BEX1 in regulating cellular homeostasis. At a low cell density, BEX1 localizes to nucleoli, where BEX1 contributes to the formation of DFCs. At a high cell density, BEX1 localizes to the apical side of cells and contributes to the formation of apical microtubules and primary cilia. $* * * p<0.001$; Student's $t$ test. The data are presented as the means \pm standard deviations
} 
a Cytoplasmic granules formed by EGFP-Bex 1
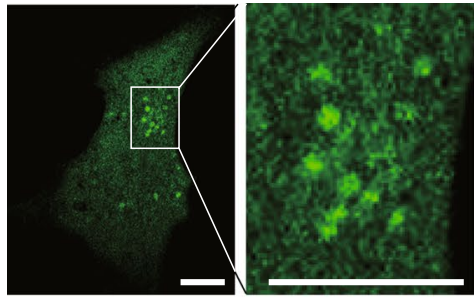

C 2D-NMR spectroscopy of ${ }^{15} \mathrm{~N}$-labeled Bex1 protein in the presence of GTP

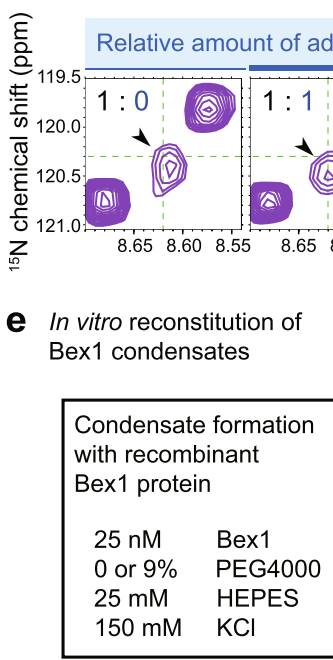

h In vitro reconstitution of Bex1 condensate-dependent tubulin polymerization

\begin{tabular}{|ll|}
\hline \multicolumn{2}{|c|}{ Tubulin polymerization } \\
with Bex1 & condensates \\
$25 \mathrm{nM}$ & Bex1 \\
$2.5 \mu \mathrm{M}$ & tubulin \\
$9 \%$ & PEG4000 \\
$25 \mathrm{mM}$ & HEPES \\
$150 \mathrm{mM}$ & KCl \\
$2 \mathrm{mM}$ & GTP \\
\hline
\end{tabular}

j Length distribution of polymerized tubulin

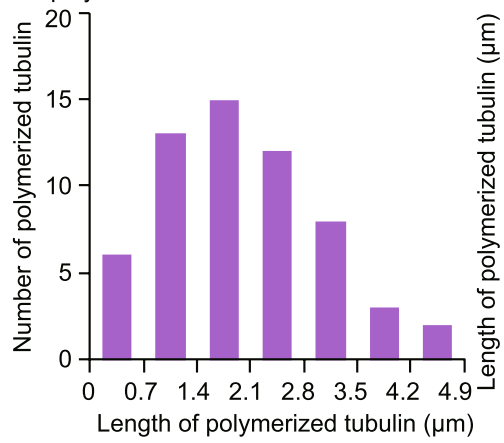

k Length of b Colocalization of EGFP-Bex1 and $\alpha$-tubulin

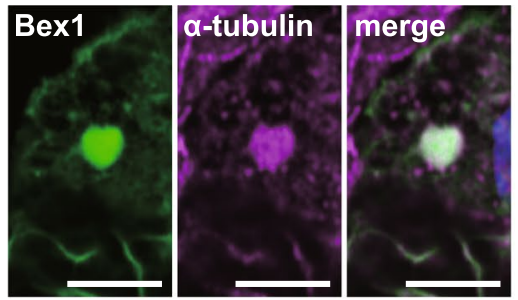

d NMR titration experiments

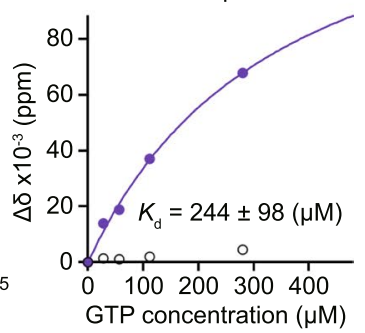

${ }^{1} \mathrm{H}$ chemical shift (ppm)

g Magnification of a Bex1 condensate ex1 condensates

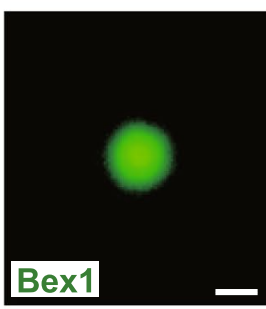

i Tubulin polymerization in the presence of Bex1 condensates

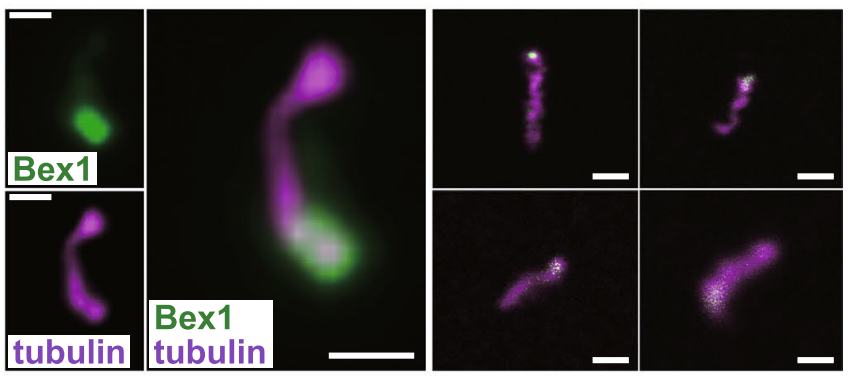

I Schematic

polymerized tubulin
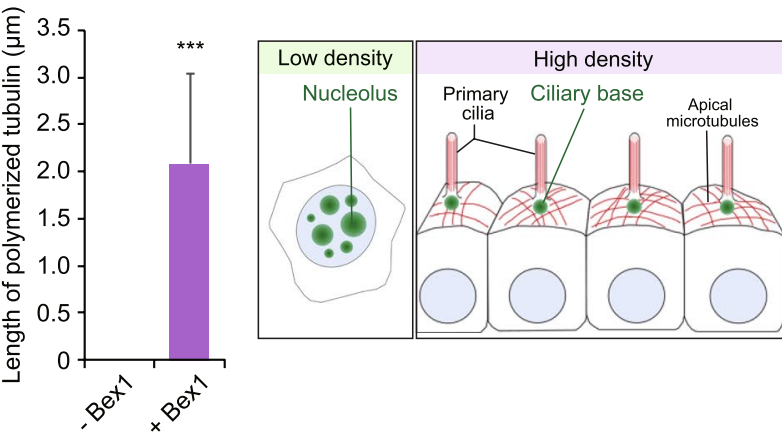

Fig. 7 (See legend on previous page.) 
compromised the primary cilia in the RPE and the striatum, but not motile cilia in the airway epithelium, indicating an essential role specific to primary cilia. Consistent with this, Bex1 mutant mice displayed ciliopathy phenotypes. In the retina, the Bex 1 mutation resulted in disoriented epithelial structures (Fig. 5G), which was in line with the in vitro findings in BEX1-depleted ARPE19 cells (Fig. 3C). Cerebellar vermis hypoplasia was previously explained by the impaired growth capacity of cerebellar granule cells due to ciliary defects $[53,54]$.

Bex 1 mutant mice showed sex differences, with female mutant mice $\left(X^{\text {mutant }} / \mathrm{X}^{\text {mutant }}\right)$ presenting more severe phenotypes than male mice $\left(X^{\text {mutant/}} Y\right)$. We therefore performed more detailed phenotypic analyses in female mice. The sex-dependent variability of the phenotypes may explain why previous research on Bex1 mutant mice did not identify ciliopathy phenotypes. Koo et al. specifically analysed male mice [5], and Accornero et al. performed cardiac pressure overload experiments in male mice [6]. The mechanism underlying this sex difference remains to be demonstrated. Further comparisons between the sexes will advance our understanding of the sex-dependent biological function of Bex1. Karp et al. performed an extensive analysis of sex differences in 14,250 WT and 40,192 mutant mice from 2,186 knockout lines of the International Mouse Phenotyping Consortium (IMPC) and showed that $56.6 \%$ of the datasets included sex differences, among which $26.6 \%$ of the phenotypes were stronger in females. Notably, half of the datasets showed stronger "eye" phenotypes in females [55]. The molecular mechanisms underlying these sex differences are still elusive, although they hold potential from biological and clinical viewpoints.

BEX1 colocalized to apical microtubules in ARPE19 and MDCK cells. BEX1 depletion abrogated apical microtubules and induced the loss of epithelial properties, which manifested in cell scattering and abnormal cell stratification. Microtubule cytoskeleton in cytosol was not affected appreciably by BEX1 knockdown. The frequent association of BEX1 with tubulin-enriched cell structures led us to find the accumulation of BEX1 in the midbody. BEX1 depletion prevented cytokinesis and caused abscission failure in ARPE19 cells and increased the number of centrosomes in NIH3T3 cells. The supernumerary centrosomes underlie the formation of supernumerary cilia, which is mediated by the compensatory role of Bex2. Bex1 depletion did not cause loss of PCNT signals, suggesting centrosomes are formed in these cells. Because Bex1 binds GTP and promote microtubule polymerization in the reconstituted system, we consider that Bex1 contributes to the maturation of the centrosomes by providing an efficient reaction field that enriches GTP for promoting the microtubule polymerization. Different dependency on Bexl was seen in ARPE19 and NIH3T3 cells. Whereas BEX1 depletion led to nearly complete loss of cilia in ARPE19 cells, Bex 1 knockdown provoked supernumerary cilia in some (9.1\%) of NIH3T3 cells. The relevance of this finding in vivo might be the tissue selectivity of the manifestation of Bex1 mutant mice. Bex 1 mutation led to the pathogenesis in the retina, cerebellum and kidney. The different dependency on Bex1 might underly the tissue-specific manifestation observed in mice.

Bex1 localizes to nucleoli at low cell density. Bex1 does not possess a nuclear localization signal (NLS) or a nucleolar localization signal (NoLS). The biological relevance of the nucleolar localization of Bex1 at low cell density has not been elucidated, but we speculate that Bex1 might interact with GTP, substrates for RNA synthesis, in nucleoli. Compared to the cells at high density, cells at low density are dividing more rigorously and tend to develop prominent nucleoli. Bex1 might contribute to the RNA synthetic function in nucleoli, which will be investigated in a future study.

To reveal the molecular function of Bex1, we adopted the unbiased approach of starting the study by analysing its amino acid sequence. This clarified the characteristics of the Bex1 protein, which did not contain any annotated domains, indicating a new principle of action. The detailed sequence analysis identified an IDR in the Bex1 amino acid sequence. For the chemical analyses, we carefully avoided any denaturing steps to allow us to investigate the native state of the Bex 1 protein in the assays. The preceding work by Fernandez et al. involved denaturing steps including boiling and measurements under acidic ( $\mathrm{pH}$ 5.1) conditions [34], thus hampering the investigation of the physiological properties of Bex1. Measurements performed at $\mathrm{pH} 7.3$ and at different temperatures, including $37^{\circ} \mathrm{C}$, verified the absence of stable secondary structures in Bex1, demonstrating that Bex1 is an IDP. Consistent with this notion, the recombinant Bex1 protein exhibited the capacity to undergo condensate formation in the presence of a molecular crowding agent.

We also investigated the interaction of Bex1 with GTP, an essential substrate for tubulin to catalyse in the polymerization reaction. The 2D-NMR spectroscopy revealed the dose-dependent peak shifts of Bex1 in the presence of GTP. The 2D-NMR analyses revealed that the chemical exchange between Bex1 and ATP was slower than that between Bex1 and GTP, implying the preferential binding of Bex1 to GTP. The colocalization of EGFPBex1 with $\alpha$-tubulin implied that Bex1 might interact with $\alpha$-tubulin, but we thus far have not detected the direct interaction of Bex1 with tubulin. On the basis of the advantages of nondenatured recombinant Bex1 purification method, the sufficiency of the Bex1 protein for 
inducing tubulin polymerization was assessed in tubulin polymerization assays. Woodruff et al. induced robust microtubule polymerization by incubating SPD-5 condensates with TPXL-1 and ZYG-9 [29]. The microtubule polymerized in the presence of Bex1 condensates appear wiggled and thinner compared to the microtubule generated in the presence of SPD-5/TPXL-1/ZYG-9. Because SPD-5 and ZYG-9 induced the microtubule polymerization more infrequently, addition of another factor to Bex1 condensates might facilitate the microtubule polymerization. Liquid-like behaviours of ciliary bases have previously been suggested in motile cilia [56] but not yet in primary cilia. Our findings implied that Bex1 forms subcellular compartments at tubulin-enriched sites and ciliary bases, where Bex1 associates with the microtubule polymerization substrates.

We focused on juvenile-expressed genes with the aim of revealing a mechanism that differentiates juveniles from adults. The study led us to identify an indispensable role of Bex 1, a JAG, in the formation of primary cilia. In the retina, the presence of primary cilia decreases as postnatal maturation continues, implying a role of Bex1 in maintaining retinal primary cilia in a specific life stage $[50,57]$. Elucidating the purpose for which primary cilia are formed only in juveniles is a goal of future work, but it is possible that cilia may sense external cues necessary to optimize tissue maturation.

Bex1 mutant mice exhibited ciliopathy phenotypes, manifested in the cornea, retina, kidney and cerebellum, forming a constellation of symptoms reminiscent of Joubert syndrome $[47,48]$. More than 35 genes are known to cause Joubert syndrome when mutated [58]. No Bex1 mutation has yet been reported to cause human diseases, but a future genetic investigation may reveal a role of the Bex1 gene in disease causation.

Different types of cilia contribute to organ development. As far as we observed, Bex 1 mutation compromises the primary cilia in the retina and striatum. The cilia of these tissues are known to be nonmotile without a central pair of microtubules (noted as " $9+0$ ",[59]). In the airway epithelium where motile $9+2$ cilia were formed, the motile cilia were not appreciably affected by Bex 1 mutation. Left-right asymmetry is introduced by motile $9+0$ cilia that produce the nodal flow. As far as we have investigated, the Bex 1 mutant mice did not exhibit randomization of left-right asymmetry (situs inversus), suggesting Bex 1 was not essential for this context. These findings indicate the different types of cilia were generated by varying machinery. Similarly, each human ciliopathy presents the variable constellation of symptoms, indicating the contribution of a particular cilia-related gene to the specific organs. Delineation of the molecular machinery underlying different types of cilia consists a future important task.

Primary cilia are functionally relevant in the retina, kidney and cerebellum. In the RPE, cilia have been reported to be necessary for the appropriate formation of retinal layers $[51,60,61]$. In the cornea, primary cilia control the proliferation of corneal epithelial [62] and endothelial [63] cells during corneal development. In the kidney, primary cilia of the renal tubular epithelium sense fluid flow and transduce mechanical signals to stimulate the cellular response of either proliferation or apoptosis [14]. Polycystic kidney disease or renal tubular malformation is another representative manifestation of ciliary defects [64]. Primary cilia were also investigated in the brains of Bex1 mutant mice. Hypoplasia of the cerebellar vermis causing hydrocephalus was observed in Bex1 mutant mice. The growth of cerebellar granule cells is known to be controlled by primary cilia $[53,54]$. Our findings in Bex 1 mutant mice will contribute to further understanding the roles of primary cilia in tissue morphogenesis.

\section{Conclusions}

In conclusion, we revealed an essential role for Bex 1 in ciliogenesis both in cells and mice. Bex1 harbours the physicochemical property as an IDP and forms biomolecular condensates which are sufficient for the induction of tubulin polymerization. In cells, Bex1 localizes to the basal body thereby providing the reaction field for the macromolecular assembly. Bex1 exhibit binding capacity to GTP that is an essential substrate for tubulin polymerization. Loss of Bex1 causes the ciliopathy phenotypes in mice thus linking the Bex1's molecular property to organogenesis. Our work focuses on the juvenile-specific molecular machinery and gives an insight into the basic principle how the mixture of molecules is built up into life.

\section{Methods \\ Establishment of Bex 1 mutant mice Reagents for genome editing}

The Bex 1 mutant mouse strains were generated using CRISPR-Cas9 technology as described previously [46]. The gRNA was designed using CRISPRdirect (https:// crispr.dbcls.jp/). The sequence of the gRNA is $5^{\prime}$-GTC GCAGGCGGTTCCGGGTTCGG-3'. The Bex2 has the identical gRNA-targeting sequence. We confirmed Bex2 was not mutated in all the strains we analysed. The gRNA sequence was cloned into the gRNA cloning vector (Addgene Plasmid ID \#41824).

For gRNA synthesis, the T7 RNA polymerase recognition site was attached to the gRNA sequence via the polymerase chain reaction (PCR). The PCR products 
were purified and used as the template for in vitro RNA synthesis using the mMESSAGE mMACHINE T7 Transcription Kit (Life Technologies). The synthesized gRNA was purified using MEGAclear (Ambion). Recombinant Cas9 protein was purchased (GeneArt Platinum Cas9 Nuclease, Thermo Scientific, B25642).

\section{Microinjection}

Mouse zygotes were obtained via in vitro fertilization (IVF) of WT C57BL6/N gametes. gRNA (100 ng/ml) and Cas 9 protein $(30 \mathrm{ng} / \mathrm{ml}$, TrueCut Cas9 Protein v2, Invitrogen) were mixed and microinjected into the pronuclei and cytoplasm of zygotes. The injected embryos were incubated at $37^{\circ} \mathrm{C}$ until they were transferred into pseudo-pregnant females at the two-cell stage.

\section{Genotyping and breeding}

Genomic DNA was extracted from the tail tips of pups, and the genomic sequence around the gRNA target site was PCR-amplified using the following primers: forward 5'-TGGCAAAAACTGTGCACCTA-3' and reverse 5'-GGTCCCCATGTCATCTTCAG-3'. The PCR products were treated with ExoSAP-IT (Thermo Fisher Scientific) and sequenced with the forward primer. For the analyses of Bex 1 mutant mice, at least 4 different strains were analysed and gave rise to similar observations. In this paper, only the $\Delta 7$ strain was used for the analyses. The female at the F1 generation having confirmed mutation was first crossed to $\mathrm{C} 57 \mathrm{BL} / 6 \mathrm{~N}$ male mice to refresh the Y chromosome. The F2 mutant male mice were then crossed with $\mathrm{C} 57 \mathrm{BL} / 6 \mathrm{~N}$ female mice to refresh the $\mathrm{X}$ chromosome and mitochondrial genome for more than 8 generations. The Bex1 mutant allele of the strain harbouring 7-nucleotide deletion we mainly described in this paper was deposited at Mouse Genome Informatics with the allele number Bex1<em1Mori> MGI:6509427.

\section{Expression analysis of Bex family genes}

The expression of Bex family genes was analysed utilizing the datasets we deposited with the accession number DRA007101 in the DDBJ Sequence Read Archive (DRA). Prenatal (embryonic day E18.5) and postnatal (postnatal day P1, P7, P14 and P56) gene expression in C57BL/6N mice $(n=3)$ were processed using Bio-Linux 8 [65] to examine the expression levels in FPKM values. Heatmaps were generated using MeV (http://mev.tm4.org).

\section{Cell culture}

ARPE19 human retinal pigment epithelial cells were cultured in DMEM:F12 medium containing 10\% foetal bovine serum (FBS) and penicillin/streptomycin. Neuro2a cells were cultured in Eagle's minimal essential medium supplemented with non-essential amino acids, $10 \%$ FBS and penicillin/streptomycin. NIH3T3 cells were cultured in DMEM containing 10\% FBS and penicillin/ streptomycin. In the knockdown experiments, Lipofectamine RNAiMAX (Invitrogen) was used to transfect the siRNA duplexes at $10 \mathrm{nM}$ : human BEX1 siRNA (Sigma-Aldrich, siRNA IDs: SASI_Hs01_00052058, SASI_Hs01_00052059, SASI_Hs01_00052061), mouse Bex1 siRNA (Bioneer, AccuTarget siRNA ID1330657), mouse Bex2 siRNA (Sigma-Aldrich, siRNA ID SASI Mm01_00072434) and siRNA negative control (Applied Biosystems, AM4611). The knockdown efficiency was assessed $48 \mathrm{~h}$ after transfection. The cellular growth was assessed by counting the number of cells $72 \mathrm{~h}$ after transfection. The phase-contrast images of the cells were taken with an EVOS FL microscope (Thermo Fisher Scientific).

\section{Gene expression analysis (qPCR)}

The total RNA was extracted using TRIzol reagent (Thermo Fisher Scientific) from cultured cells according to the manufacturer's instruction. The extracted RNA was quantified using a NanoDrop Lite Spectrophotometer (Thermo Fisher Scientific) and reverse transcribed using a High-Capacity RNA-to-cDNA Kit (Applied Biosystems) and a PCR Thermal Cycler Dice (Takara) according to the manufacturer's instructions. qPCR was performed using a LightCycler 480 SYBR Green I Master Kit on a LightCycler 480 instrument (Roche) using the reverse-transcribed cDNA as a template. The specificity and quality of the qPCR amplification was assessed by performing a melting curve analysis. The data were normalized to human $T U B B$ or mouse Tubb5 as indicated in the Figure legends. The sequences of the primers are as follows: mouse Bex1 5'-GGAGCAGGTCTGAGAAGC AG-3' and 5'-CACGCCTTGATCTTTGGACT-3'; mouse Bex2 5'-TGACTGGAAACCGAGAGTCC-3 ${ }^{\prime}$ and $5^{\prime}-\mathrm{CCT}$ CCTTTTCCTGATGGTCA-3'; mouse Tubb5 5'-GAT CGGTGCTAAGTTCTGGGA-3' and 5'-AGGGACATA CTTGCCACCTGT-3'; human BEX1 5'-AGAATCGGG AGAAGGAGGAG- $3^{\prime}$ and $5^{\prime}$-TTTCTTGGTTGGCAT TTTCC-3'; human TUBB 5'-TGGACTCTGTTCGCT CAGGT-3' ${ }^{\prime}$ and $5^{\prime}$-TGCCTCCTTCCGTACCACAT- ${ }^{\prime}$.

\section{Immunocytochemistry}

ARPE19 cells, Neuro2a and NIH3T3 cells were fixed with $4 \%$ paraformaldehyde (PFA) for $10 \mathrm{~min}$ at room temperature (RT), permeabilized with $0.1 \%$ Triton X-100 for 2 min, blocked with 2\% FBS at RT and incubated with antibody against Bex1 (ProteinTech, 12390-1-AP, 1:100), $\alpha$-catenin (CST, \#3240, 1:200), $\beta$-catenin (CST, \#8480, 1:200), Vimentin (Santa Cruz, sc-6260, 1:100), 
Filamin (Thermo Scientific, \#MS-1211, 1:40), Fibrillarin (FBL, 38F3, Novus, \#NB300-269, 1:500), Nucleolin (CST, \#87792S, 1:400), ARL13B (Abcam, ab1366481:100), acetylated tubulin (Sigma, T6793, 1:500), IFT88 (ProteinTech, 13967-1-AP, 1:100), PCNT (Abcam, ab4448, 1:500), PCNT-Alexa 594 (Novus, NB100-61071AF594, 1:200), LIN28B (CST, \#4196S, 1:200), G3BP1 (Bethyl, A302033A, 1:500) and tubulin (CST, \#2148, 1:200) at $4^{\circ} \mathrm{C}$ overnight. After washing with PBS for 10 min 3 times, cells were incubated with appropriate secondary antibodies anti-rabbit-Alexa Fluor 488, anti-mouse-Alexa Fluor 594 or anti-mouse-Alexa Fluor 647 (1:1,000, Invitrogen) at RT for $1 \mathrm{~h}$. Slides were mounted with Prolong Gold with DAPI (Invitrogen). Images were taken by confocal microscope TCS SP8 (Leica). For the analysis of the primary cilia, the frequency of ciliated cells and the number of cilia per cell were counted. Because a cell density or cell cycle status can influence ciliogenesis, the analysis of cilia was performed at similar cell density both for the normal and the Bex1-depleted conditions. The length of the primary cilia was measured with Leica Application Suite X software. The sphericity of nuclei was calculated with the images of Fibrillarin immunostaining, using the circularity plugin of the NIH ImageJ Fiji [66] using the formula: circularity $=4 \pi\left(\right.$ area $/$ perimeter $\left.^{2}\right)$.

\section{Western blot analysis with mouse tissues}

For sampling mouse tissues, dissected tissues were crushed using a homogenizer ( $\mu \mathrm{T}-12$, Taitec) in chilled RIPA buffer containing $25 \mathrm{mM}$ Tris- $\mathrm{HCl}$ (pH 7.6), 150 $\mathrm{mM} \mathrm{NaCl}, 1 \% \mathrm{NP}-40,1 \%$ sodium deoxycholate and $0.1 \%$ SDS. The lysates were centrifuged at $20,400 \times g$ for $10 \mathrm{~min}$ to remove debris. Laemmli sample buffer was added to the lysates, followed by boiling at $95^{\circ} \mathrm{C}$ for $2 \mathrm{~min}$. The protein samples $(10 \mu \mathrm{g}$ per lane) were separated by SDSPAGE and transferred to a Hybond-P PVDF membrane (GE Healthcare). Because an antibody reactive to mouse Bex1 protein was not commercially available, we developed a new antibody reactive to mouse Bex1. Polyclonal antisera were generated by immunizing rabbits with the peptide $\left[\mathrm{NH}_{2}-\mathrm{C}+\mathrm{KKEEKEEKPQDTIR-COOH}\right]$ derived from the Bex1-specific amino acid sequence. The $\mathrm{N}$ terminal cysteine $[\mathrm{C}+]$ of the peptide was added for the purpose of conjugating the peptide to carrier protein. As loading control, alpha-tubulin (1:4000, Cell Signaling Technology, \#3873) and Gapdh (1:4000, Cell Signaling Technology, \#5174) were used. Secondary antibodies conjugated with horseradish peroxidase (Thermo Fisher Scientific, anti-mouse 32430 and anti-rabbit 32460) were used at 1:1000 dilution. The immunoreactive bands were detected using Chemi-Lumi One L or Chemi-Lumi One Ultra (Nacalai Tesque, Kyoto, Japan).

\section{Mouse histological analysis}

The ocular histology was analysed by the ophthalmologists. Following the euthanization of the mice, the eyes were enucleated and were fixed with 4\% PFA at RT for $30 \mathrm{~min}$. For haematoxylin and eosin (HE) staining, samples were paraffin-embedded and sectioned at $3 \mu \mathrm{m}$ thickness. The images were taken by Nikon ECLIPSE 90i (Nikon). For immunohistochemistry, after removal of the optic nerve, cornea and lens, the retina was cut into quadrants, making angles at 90 degrees to the optic nerve head at the centre. The flat-mounted retina was analysed by immunohistochemistry as described below.

For the histological analysis in brains and kidneys, the mice were systemically fixed with $4 \%$ PFA followed by dissection of the organs. The dissected organs were postfixed with $4 \%$ PFA for $3 \mathrm{~h}$ followed by sectioning at $3 \mu \mathrm{m}$ thickness. The tissue sections were stained with HE. The images were taken by NanoZoomer S210 slide scanner (Hamamatsu) and analysed with NanoZoomer U1238801 software (Hamamatsu). Histological diagnosis was made by the nephrologist.

For the immunohistochemistry with the retina and striatum, the flat-mounted retina or the PFA-fixed brain was permeabilized with $0.1 \%$ Triton X-100 for 2 min and blocked with $2 \% \mathrm{FBS}$ at RT for $60 \mathrm{~min}$. The tissues were incubated with the primary antibody against acetylated tubulin (Sigma, T6793, 1:500) at $4^{\circ} \mathrm{C}$ overnight. After washing with phosphate buffer saline (PBS), the tissues were incubated with the secondary antibody anti-mouseAlexa Fluor 594 (1:1,000, Invitrogen). After washing with PBS, the tissues were mounted with Prolong Gold with DAPI (Invitrogen). Images were taken by confocal microscope TCS SP8 (Leica). For the analysis of the primary cilia, the frequency of ciliated cells was counted. The length of the primary cilia was measured with Leica Application Suite X software.

\section{Protein expression and purification}

The expression plasmid for mouse Bex1 were constructed with fusion proteins tagged with hexahistidine (His) at the $\mathrm{N}$-terminus by inserting Bex 1 into the pETDuet-1 vector. The pETDuet-1-Bex1 vector was introduced into the E. coli strain Rosetta-gami 2(DE3) pLysS. Transformed bacteria were grown in Luria broth medium containing ampicillin. Uniformly ${ }^{15} \mathrm{~N}$-labelled proteins were grown in M9 minimal medium with $0.5 \mathrm{~g} / \mathrm{L}{ }^{15} \mathrm{NH}_{4} \mathrm{Cl}$. Bacterial cells were grown to an $\mathrm{OD}_{600}$ of $\sim 0.6$ at $37^{\circ} \mathrm{C}$, and then isopropyl- $\beta-\mathrm{D}$ thiogalactopyranoside (IPTG) was added to the medium. Cells were further incubated at $37^{\circ} \mathrm{C}$ for $5 \mathrm{~h}$ and were then harvested by centrifugation at $5000 \times g$ for $10 \mathrm{~min}$ at $4^{\circ} \mathrm{C}$. The collected bacterial cells were suspended in the 
buffer containing $20 \mathrm{mM}$ sodium phosphate ( $\mathrm{pH}$ 8.0), 1 $\mathrm{M} \mathrm{NaCl}, 0.2 \mathrm{mg} / \mathrm{mL}$ lysozyme, DNase I and $1 \mathrm{mM}$ phenylmethanesulfonyl fluoride (PMSF) and were rotated for $1 \mathrm{~h}$ at $4^{\circ} \mathrm{C}$. The cells were sonicated three cycles with intermittent pulses for $1 \mathrm{~min}$ (pulse of $0.7 \mathrm{~s}$, interval of $0.3 \mathrm{~s}$, output level of 3) using an ultrasonic disruptor equipped with a standard flat tip (Sonifier 450 Advance, Branson Ultrasonics, Connecticut). After removal of cell debris by centrifugation at $12,000 \times g$ for $45 \mathrm{~min}$ at $4^{\circ} \mathrm{C}$, the cell extract was loaded onto a Ni-NTA resin (QIAGEN). The column was washed with a solution spectrum was measured for $2.5 \mathrm{~h}$ at $25^{\circ} \mathrm{C}$. Chemical shift perturbation $(\Delta \delta)$ was calculated by the following equation (1);

$$
\Delta \delta=\sqrt{\left(\Delta \delta_{\mathrm{H}}\right)^{2}+\left(\Delta \delta_{\mathrm{N}} / 5\right)^{2}}
$$

where $\Delta \delta_{\mathrm{H}}$ and $\Delta \delta_{\mathrm{N}}$ are the chemical shift differences in the presence and absence of the GTP, respectively.

The concentration of GTP was plotted on the horizontal axis against $\Delta \delta$ on the vertical axis and fitted to obtain binding constant $\left(K_{\mathrm{d}}\right)$ by the following eq. (2);

$$
\Delta \delta_{\mathrm{H}, \mathrm{N}}=\Delta \delta_{\max } \times \frac{\left([\text { Bex } 1]+[\mathrm{GTP}]+K_{\mathrm{d}}\right)-\sqrt{\left([\mathrm{Bex} 1]+[\mathrm{GTP}]+K_{\mathrm{d}}\right)^{2}-4[\mathrm{Bex} 1] \times[\mathrm{GTP}]}}{2 \times[\operatorname{Bex} 1]}
$$

containing $20 \mathrm{mM}$ sodium phosphate ( $\mathrm{pH} 8.0), 300 \mathrm{mM}$ $\mathrm{NaCl}$ and $20 \mathrm{mM}$ imidazole, followed by the elution with the buffer containing $20 \mathrm{mM}$ sodium phosphate $(\mathrm{pH}$ 8.0), $300 \mathrm{mM} \mathrm{NaCl}$ and $500 \mathrm{mM}$ imidazole. The purity of the protein was analysed by SDS-PAGE. The purified protein was collected, dialyzed against suitable solution for each experiment and concentrated with a powder of absorbent gel (Spectra/Gel ${ }^{\circledR}$ Absorbent, Spectrum-Labs, Rancho Dominguez, CA, USA) at $4{ }^{\circ} \mathrm{C}$. The concentration of Bex1 protein was calculated from the spectra measured by NanoDrop One (Thermo Scientific), using an absorption coefficient of 7000 .

\section{CD spectroscopy}

Circular dichroism CD spectra between 195 and $250 \mathrm{~nm}$ were collected on a J-805 spectropolarimeter (JASCO, Tokyo, Japan) at 10,25 , and $37{ }^{\circ} \mathrm{C}$. We measured $200 \mu \mathrm{L}$ solution of 5-10 uM BEX1 in $20 \mathrm{mM}$ sodium phosphate buffer, $140 \mathrm{mM} \mathrm{NaCl}, \mathrm{pH} 7.3$ in a quartz cuvette with a $1 \mathrm{~mm}$ light path length. The results were expressed as apparent ellipticity with the unit of ellipticity.

\section{Nuclear magnetic resonance (NMR) experiments}

The two-dimensional ${ }^{1} \mathrm{H}_{-}{ }^{15} \mathrm{~N}$ HSQC spectrum was measured with a Bruker Avance III 900-MHz spectrometer equipped with a cryomagnetic probe. NMR experiments were performed at the protein concentration of $28 \mu \mathrm{M}$. The solvent conditions were $20 \mathrm{mM}$ sodium phosphate buffer, $140 \mathrm{mM} \mathrm{NaCl}, 5 \% \mathrm{D}_{2} \mathrm{O}, \mathrm{pH} 7.3$. The chemical shift values were referenced to 4,4-dimethyl-4-silapentane1-sulfonic acid (DSS).

In the NMR titration experiment with Bex1 and GTP, GTP was added to $28 \mu \mathrm{M}$ Bex 1 at concentrations of 28 $\mu \mathrm{M}, 56 \mu \mathrm{M}, 112 \mu \mathrm{M}$ and $280 \mu \mathrm{M}$, and each ${ }^{1} \mathrm{H}_{-}{ }^{15} \mathrm{~N}$ HSQC where $\Delta \delta_{\max }$ is $\Delta \delta_{\mathrm{H}, \mathrm{N}}$ in case of moving to the maximum, and $\Delta \delta_{\max }$ and $K_{\mathrm{d}}$ were calculated by fitting Eq. (2) using Igor application.

\section{Structural modelling}

The tertiary structures of Bex family proteins were assessed by Phyre2 (http://www.sbg.bio.ic.ac.uk/phyre2/ $\mathrm{html} /$ page.cgi?id=index). The property of Bex family proteins as intrinsically disordered proteins (IDPs) was assessed by PrDOS (http://prdos.hgc.jp/) and DISOPRED3 (http://bioinf.cs.ucl.ac.uk/psipred/).

\section{In vitro reconstitution assays}

The Bex1 condensate formation was tested by the method described previously [32]. The recombinant Bex1 protein $(10 \mu \mathrm{M})$ was labelled with DyLight 594 NHS ester according to the manufacturer's instruction (ThermoFisher Scientific). To remove unbound dye, dialysis was performed with the dialysis buffer containing $25 \mathrm{mM}$ HEPES and $150 \mathrm{mM} \mathrm{KCl}$ using BioDesignDialysis Tubing (molecular weight cut off $=8000 \mathrm{Da}$, BioDesign Inc. of New York). The labelled Bex1 protein (final concentration $=25 \mathrm{nM}$ ) was diluted in the buffer containing 25 mM HEPES, $150 \mathrm{mM} \mathrm{KCl,} 0.5 \mathrm{mM}$ dithiothreitol (DTT) in the presence $(9 \%)$ or absence $(0 \%)$ of polyethylene glycol (PEG) 4000 and incubated for $5 \mathrm{~min}$ at $25^{\circ} \mathrm{C}$. Images of the Bex1 condensates were taken by IX83 inverted microscope (Olympus). Pseudo-coloured images were generated to show Bex1 signals in green.

The potency of the Bex1 condensates to promote tubulin polymerization was assessed by incubating the Bex1 condensates with tubulin protein. For the tubulin, Alexa 488-labelled (catalogue number: 048805, PurSolutions, Tennessee) or unlabelled (catalogue number: 032005) 
tubulin was purchased and mixed at 1:4. The Bex1 condensates were incubated with the mixed tubulin at 2.5 $\mu \mathrm{M}$ in the buffer containing $25 \mathrm{mM}$ HEPES, $150 \mathrm{mM} \mathrm{KCl}$, $0.5 \mathrm{mM}$ DTT, 9\% PEG and $2 \mathrm{mM}$ GTP and incubated for $10 \mathrm{~min}$ at $25^{\circ} \mathrm{C}$. Images of polymerized tubulin were obtained by IX83 inverted microscope (Olympus) and confocal microscope TCS SP8 (Leica). Pseudo-coloured images were generated to show Bex1 signals in green and tubulin signals in magenta.

\section{Fluorescent recovery after photo-bleaching (FRAP)}

For the in vivo experiments, FRAP of EGFP-Bex1 in ARPE19 cells was performed on Fluoview FV3000 confocal microscope system (Olympus). Using a $60 \times$ oil immersion objective, a whole EGFP-Bex1 granule was bleached using a laser intensity of $20 \%$ at $488 \mathrm{~nm}$. Recovery was recorded for every second for a total of $300 \mathrm{~s}$ after bleaching. Analysis of the recovery curves was carried out with cellSens software (Olympus).

For the in vitro experiments, FRAP was carried out with samples in glass bottom 8-well chamber slides using a Fluoview FV3000 confocal microscope equipped with $60 \times$ oil immersion objectives, as above. Condensates were bleached using a laser intensity of $30 \%$ at $561 \mathrm{~nm}$. Recovery was recorded for every second for a total of $180 \mathrm{~s}$ after bleaching. Analysis of the recovery curves was carried out with cellSens software (Olympus).

\section{Statistical analysis}

For all quantified data, mean \pm standard deviation (SD) was presented. Statistical significance between two experimental groups was indicated by an asterisk and comparisons were made using the Student's $t$ test. $P$ values less than 0.05 were considered significant.

\footnotetext{
Abbreviations

ATP: Adenosine triphosphate; CD: Circular dichroism; DFC: Dense fibrillar component; FBL: Fibrillarin; FRAP: Fluorescence recovery after photobleaching; GC: Granular component; GTP: Guanosine triphosphate; IDP: Intrinsically disordered protein; IDR: Intrinsically disordered region; JAGs: Juvenility-associated genes; KO: Knockout; NMR: Nuclear magnetic resonance; PCM: Pericentriolar matrix; PEG: Polyethylene glycol; RPE: Retinal pigment epithelium; WT: Wild type.
}

\section{Supplementary Information}

The online version contains supplementary material available at https://doi. org/10.1186/s12915-022-01246-x.

\section{Additional file 1.}

\section{Acknowledgements}

We would like to thank all the lab members from the National Cerebral and Cardiovascular Centre Research Institute for their helpful discussions and sincere cooperation. We are grateful to Dr. James Pearson for critical reading of the manuscript. This study was supported by the Central Research Laboratory at Shiga University of medical science (SUMS).

\section{Authors' contributions}

E.H. and M.M. performed the experiments with the recombinant proteins. Y.I. performed ocular analyses. A.Ts. and M.M. performed mouse experiments and histological analyses. Y.S. performed the imaging analyses of molecular condensates. T.M., M.O. Y.M. and M.N. assisted in the data analysis and discussed the results with M.M. E.H. and M.M conceived the experiment and wrote the manuscript. All authors read and approved the final manuscript.

\section{Funding}

M.M. is supported by research grants from the Japan Epilepsy Research Foundation (JERF), Japan Brain Foundation, Takeda Science Foundation, the Japan Spina Bifida \& Hydrocephalus Research Foundation and Kawano Masanori Memorial Public Interest Incorporated Foundation for Promotion of Paediatrics. This study was supported by Grants-in-Aid for Scientific Research for Young Scientists from the Japan Intractable Diseases (Nanbyo) Research Foundation. This study was supported by JSPS KAKENHI Grant Numbers $18 \mathrm{~K} 07788$ and 19H04774, the Leading Initiative for Excellent Young Researchers (LEADER) 5013323 and Initiative on Rare and Undiagnosed Diseases (IRUD) of AMED.

\section{Availability of data and materials}

The datasets supporting the conclusion of this article are included within the article.

\section{Declarations}

Ethics approval and consent to participate

All animal experiments were approved by the institutional animal care and use committee at the Shiga University of Medical Science and National Cerebral and Cardiovascular Centre Research Institute. All experiments were performed in accordance with the relevant guidelines and regulations.

\section{Consent for publication}

Not applicable.

\section{Competing interests}

The authors declare that they have no competing interests.

\section{Author details}

${ }^{1}$ Molecular Neuroscience Research Centre (MNRC), Shiga University of Medical Science, Seta Tsukinowa-cho, Otsu, Shiga 520-2192, Japan. ${ }^{2}$ Graduate School of Pharmaceutical Sciences, Nagoya University, Furo-cho, Chikusa-ku, Nagoya 464-8601, Japan. ${ }^{3}$ Department of Ophthalmology, Shiga University of Medical Science, Seta Tsukinowa-cho, Otsu, Shiga 520-2192, Japan. ${ }^{4}$ Department of Paediatrics, Shiga University of Medical Science, Seta Tsukinowa-cho, Otsu, Shiga 520-2192, Japan. ${ }^{5}$ Department of Vascular Physiology, National Cerebral and Cardiovascular Centre Research Institute, 6-1 Kishibe-Shimmachi, Suita, Osaka 564-8565, Japan. ${ }^{6}$ Research Institute for Interdisciplinary Science, Okayama University, 3-1-1 Tsushima-naka, Kita-ku, Okayama 700-8530, Japan.

Received: 14 August 2021 Accepted: 2 February 2022

Published online: 10 February 2022

\section{References}

1. Jam FA, Kadota Y, Mendsaikhan A, Tooyama I, Mori M. Identification of juvenility-associated genes in the mouse hepatocytes and cardiomyocytes. Sci Rep. 2018;8:3132. https://doi.org/10.1038/s41598-018-21445-3.

2. Yang Y, Tetti M, Vohra T, Adolf C, Seissler J, Hristov M, et al. BEX1 Is Differentially Expressed in Aldosterone-Producing Adenomas and Protects Human Adrenocortical Cells from Ferroptosis. Hypertension. 2021; May:1647-58.

3. Wang Q, Liang N, Yang T, Li Y, Li J, Huang Q, et al. DNMT1-mediated methylation of BEX1 regulates stemness and tumorigenicity in liver cancer. J Hepatol. 2021;75:1142-53. https://doi.org/10.1016/j.jhep.2021.06.025. 
4. Doi T, Ogawa H, Tanaka Y, Hayashi Y, Maniwa Y. <em>Bex $1</$ em > significantly contributes to the proliferation and invasiveness of malignant tumor cells. Oncol Lett. 2020;20:1-1. https://doi.org/10.3892/ol.2020. 12226.

5. Koo JH, Smiley MA, Lovering RM, Margolis FL. Bex 1 knock out mice show altered skeletal muscle regeneration. Biochem Biophys Res Commun. 2007;363:405-10.

6. Accornero F, Schips TG, Petrosino JM, Gu S-Q, Kanisicak O, van Berlo JH, et al. BEX1 is an RNA-dependent mediator of cardiomyopathy. Nat Commun. 2017:8:1875. https://doi.org/10.1038/s41467-017-02005-1.

7. Nonaka S, Tanaka Y, Okada Y, Takeda S, Harada A, Kanai Y, et al. Randomization of Left-Right Asymmetry due to Loss of Nodal Cilia Generating Leftward Flow of Extraembryonic Fluid in Mice Lacking KIF3B Motor Protein. Cell. 1998;95:829-37. https://doi.org/10.1016/S0092-8674(00) 81705-5.

8. Sawamoto K. New Neurons Follow the Flow of Cerebrospinal Fluid in the Adult Brain. Science. 2006;311:629-32. https://doi.org/10.1126/science. 1119133

9. Mirzadeh Z, Kusne Y, Duran-Moreno M, Cabrales E, Gil-Perotin S, Ortiz C, et al. Bi- and uniciliated ependymal cells define continuous floor-platederived tanycytic territories. Nat Commun. 2017;8:1-12. https://doi.org/ 10.1038/ncomms 13759 .

10. Oh EC, Katsanis N. Cilia in vertebrate development and disease. Development. 2012;139:443-8. https://doi.org/10.1242/dev.050054.

11. Malicki JJ, Johnson CA. The Cilium: Cellular Antenna and Central Processing Unit. Trends Cell Biol. 2017;27:126-40. https://doi.org/10.1016/j.tcb. 2016.08.002.

12. Singla V, Reiter JF. The primary cilium as the cell's antenna: Signaling at a sensory organelle. Science. 2006;313:629-33.

13. Berbari NF, O'Connor AK, Haycraft CJ, Yoder BK. The Primary Cilium as a Complex Signaling Center. Curr Biol. 2009;19:R526-35. https://doi.org/10. 1016/j.cub.2009.05.025.

14. Guay-Woodford LM. Renal cystic diseases: Diverse phenotypes converge on the cilium/centrosome complex. Pediatr Nephrol. 2006;21:1369-76.

15. ParkTJ, Haigo SL, Wallingford JB. Ciliogenesis defects in embryos lacking inturned or fuzzy function are associated with failure of planar cell polarity and Hedgehog signaling. Nat Genet. 2006;38:303-11.

16. Kim SK, Shindo A, Park TJ, Oh EC, Ghosh S, Gray RS, et al. Planar Cell Polarity Acts Through Septins to Control Collective Cell Movement and Ciliogenesis. Science. 2010;329:1337-40. https://doi.org/10.1126/science. 1191184.

17. Han Y, Kim HJ, Dlugosz AA, Ellison DW, Gilbertson RJ, Alvarez-Buylla A. Dual and opposing roles of primary cilia in medulloblastoma development. Nat Med. 2009;15:1062-5. https://doi.org/10.1038/nm.2020.

18. Reiter JF, Leroux MR. Genes and molecular pathways underpinning ciliopathies. Nat Rev Mol Cell Biol. 2017;18:533-47. https://doi.org/10.1038/ nrm.2017.60.

19. Mitchison HM, Valente EM. Motile and non-motile cilia in human pathology: from function to phenotypes. J Pathol. 2017;241:294-309.

20. Parisi MA. Clinical and molecular features of Joubert syndrome and related disorders. Am J Med Genet Part C Semin Med Genet. 2009;151:326-40.

21. Brancati F, Dallapiccola B, Valente E. Joubert Syndrome and related disorders. Orphanet J Rare Dis. 2010;5:20. https://doi.org/10.1186/ 1750-1172-5-20.

22. Vertii A, Hung HF, Hehnly H, Doxsey S. Human basal body basics. Cilia. 2016:5:1-7.

23. Winey M, O'Toole E. Centriole structure. Philos Trans R Soc B Biol Sci. 2014;369.

24. Mennella V, Agard DA, Huang B, Pelletier L. Amorphous no more: Subdiffraction view of the pericentriolar material architecture. Trends Cell Biol. 2014;24:188-97. https://doi.org/10.1016/j.tcb.2013.10.001.

25. Doxsey SJ, Stein P, Evans L, Calarco PD, Kirschner M. Pericentrin, a highly conserved centrosome protein involved in microtubule organization. Cell. 1994;76:639-50. https://doi.org/10.1016/0092-8674(94)90504-5.

26. Woodruff JB, Wueseke $\mathrm{O}$, Hyman AA. Pericentriolar material structure and dynamics. Philos Trans R Soc B Biol Sci. 2014;369.

27. David-Pfeuty T, Erickson HP, Pantaloni D. Guanosinetriphosphatase activity of tubulin associated with microtubule assembly. Proc Natl Acad Sci U S A. 1977;74:5372-6.
28. Babu MM, van der Lee R, de Groot NS, Gsponer J. Intrinsically disordered proteins: Regulation and disease. Curr Opin Struct Biol. 2011;21:432-40.

29. van der Lee R, Buljan M, Lang B, Weatheritt RJ, Daughdrill GW, Dunker AK, et al. Classification of Intrinsically Disordered Regions and Proteins. Chem Rev. 2014;114:6589-631. https://doi.org/10.1021/cr400525m.

30. Wright PE, Dyson $\mathrm{HJ}$. Intrinsically disordered proteins in cellular signalling and regulation. Nat Rev Mol Cell Biol. 2015;16:18-29. https://doi.org/10. 1038/nrm3920

31. Banani SF, Lee HO, Hyman AA, Rosen MK. Biomolecular condensates: Organizers of cellular biochemistry. Nat Rev Mol Cell Biol. 2017;18:285-98. https://doi.org/10.1038/nrm.2017.7.

32. Woodruff JB, Ferreira Gomes B, Widlund PO, Mahamid J, Honigmann A, Hyman AA. The Centrosome Is a Selective Condensate that Nucleates Microtubules by Concentrating Tubulin. Cell. 2017;169:1066-1077.e10. doi:10.1016/j.cell.2017.05.028

33. Kelley LA, Mezulis S, Yates CM, Wass MN, Sternberg MJE. The Phyre2 web portal for protein modeling, prediction and analysis. Nat Protoc. 2015;10:845-58. https://doi.org/10.1038/nprot.2015.053.

34. Fernandez EM, Díaz-Ceso MD, Vilar M. Brain Expressed and X-Linked (Bex) Proteins Are Intrinsically Disordered Proteins (IDPs) and Form New Signaling Hubs. PLoS One. 2015;10:e0117206. https://doi.org/10.1371/journal. pone.0117206.

35. Hiramatsu N, Hibino E, Matsuzaki K, Kuwahara J, Hoshino M. Interaction between isolated transcriptional activation domains of Sp1 revealed by heteronuclear magnetic resonance. Protein Sci. 2012;21:1481-8.

36. Hibino E, Inoue R, Sugiyama M, Kuwahara J, Matsuzaki K, Hoshino M. Identification of heteromolecular binding sites in transcription factors Sp1 and TAF4 using high-resolution nuclear magnetic resonance spectroscopy. Protein Sci. 2017;26:2280-90. https://doi.org/10.1002/ pro.3287

37. Wishart DS, Bigam CG, Holm A, Hodges RS, Sykes BD. $1 \mathrm{H}, 13 \mathrm{C}$ and $15 \mathrm{~N}$ random coil NMR chemical shifts of the common amino acids. I. Investigations of nearest-neighbor effects. J Biomol NMR. 1995;5:67-81.

38. Wei T, Baiqu H, Chunxiang L, Zhonghe Z. In situ visualization of rDNA arrangement and its relationship with subnucleolar structural regions in Allium sativum cell nucleolus. J Cell Sci. 2003;116:1117-25. https://doi. org/10.1242/jcs.00323.

39. Boisvert FM, Van Koningsbruggen S, Navascués J, Lamond Al. The multifunctional nucleolus. Nat Rev Mol Cell Biol. 2007;8:574-85.

40. He J-S, Soo P, Evers M, Parsons KM, Hein N, Hannan KM, et al. HighContent Imaging Approaches to Quantitate Stress-Induced Changes in Nucleolar Morphology. Assay Drug Dev Technol. 2018;16:320-32. https:// doi.org/10.1089/adt.2018.861.

41. Choi K, Lee K, Ryu SW, Im M, Kook KH, Choi C. Pirfenidone inhibits transforming growth factor- $\beta 1$-induced fibrogenesis by blocking nuclear translocation of smads in human retinal pigment epithelial cell line ARPE19. Mol Vis. 2011:2012(18):1010-20.

42. Lee J, Choi JH, Joo CK. TGF- $\beta 1$ regulates cell fate during epithelial-mesenchymal transition by upregulating survivin. Cell Death Dis. 2013;4:1-10.

43. Li H, Wang $H$, Wang $F, G u Q, X u X$. Snail involves in the transforming growth factor $\beta 1$-mediated epithelial-mesenchymal transition of retinal pigment epithelial cells. PLoS One. 2011;6.

44. Cong L, Ran FA, Cox D, Lin S, Barretto R, Habib N, et al. Multiplex Genome Engineering Using CRISPR/Cas Systems. Science. 2013;339:819-23. https://doi.org/10.1126/science.1231143.

45. Mali P, Yang L, Esvelt KM, Aach J, Guell M, DiCarlo JE, et al. RNA-Guided Human Genome Engineering via Cas9. Science. 2013;339:823-6. https:// doi.org/10.1126/science.1232033.

46. Inui M, Miyado M, Igarashi M, Tamano M, Kubo A, Yamashita S, et al. Rapid generation of mouse models with defined point mutations by the CRISPR/Cas9 system. Sci Rep. 2015;4:5396. https://doi.org/10.1038/srep0 5396.

47. Waters AM, Beales PL. Ciliopathies: an expanding disease spectrum. Pediatr Nephrol. 2011;26:1039-56. https://doi.org/10.1007/ s00467-010-1731-7.

48. Hildebrandt F, Benzing T, Katsanis N. Ciliopathies. N Engl J Med. 2011;364:1533-43. https://doi.org/10.1056/NEJMra1010172.

49. Simms RJ, Hynes AM, Eley L, Sayer JA. Nephronophthisis: A Genetically Diverse Ciliopathy. Int J Nephrol. 2011;2011:1-10. https://doi.org/10.4061/ 2011/527137. 
50. Nishiyama K, Sakaguchi H, Hu JG, Bok D, Hollyfield JG. Claudin localization in cilia of the retinal pigment epithelium. Anat Rec. 2002;267:196-203. https://doi.org/10.1002/ar.10102.

51. Wheway G, Parry DA, Johnson CA. The role of primary cilia in the development and disease of the retina. Organogenesis. 2014;10:69-85. https:// doi.org/10.4161/org.26710.

52. May-Simera HL, Wan Q, Jha BS, Hartford J, Khristov V, Dejene R, et al. Primary Cilium-Mediated Retinal Pigment Epithelium Maturation Is Disrupted in Ciliopathy Patient Cells. Cell Rep. 2018;22:189-205. https:// doi.org/10.1016/j.celrep.2017.12.038.

53. Chizhikov VV, Davenport J, Zhang Q, Shih EK, Cabello OA, Fuchs JL, et al. Cilia Proteins Control Cerebellar Morphogenesis by Promoting Expansion of the Granule Progenitor Pool. J Neurosci. 2007;27:9780-9. https://doi. org/10.1523/JNEUROSCI.5586-06.2007.

54. Spassky N, Han Y-G, Aguilar A, Strehl L, Besse L, Laclef C, et al. Primary cilia are required for cerebellar development and Shh-dependent expansion of progenitor pool. Dev Biol. 2008;317:246-59. https://doi.org/10.1016/j. ydbio.2008.02.026.

55. Karp NA, Mason J, Beaudet AL, Benjamini Y, Bower L, Braun RE, et al. Prevalence of sexual dimorphism in mammalian phenotypic traits. Nat Commun. 2017;8.

56. Huizar RL, Lee C, Boulgakov AA, Horani A, Tu F, Marcotte EM, et al. A liquid-like organelle at the root of motile ciliopathy. Elife. 2018;7:1-24

57. Patnaik SR. Kretschmer V, Brücker L, Schneider S, Volz AK, Oancea-Castillo $L$ del $R$, et al. Bardet-Biedl Syndrome proteins regulate cilia disassembly during tissue maturation. Cell Mol Life Sci. 2019;76:757-75. https://doi. org/10.1007/s00018-018-2966-x

58. Parisi MA. The molecular genetics of Joubert syndrome and related ciliopathies: The challenges of genetic and phenotypic heterogeneity. Transl Sci Rare Dis. 2019;4:25-49.

59. Choksi SP, Lauter G, Swoboda P, Roy S. Switching on cilia:Transcriptional networks regulating ciliogenesis. Dev. 2014;141:1427-41.

60. Bujakowska KM, Liu Q, Pierce EA. Photoreceptor Cilia and Retinal Ciliopathies. Cold Spring Harb Perspect Biol. 2017;9:a028274. https://doi.org/10 1101/cshperspect.a028274.

61. May-Simera HL, Gumerson JD, Gao C, Campos M, Cologna SM, Beyer T, et al. Loss of MACF1 Abolishes Ciliogenesis and Disrupts Apicobasal Polarity Establishment in the Retina. Cell Rep. 2016;17:1399-413. https:// doi.org/10.1016/j.celrep.2016.09.089.

62. Grisanti L, Revenkova E, Gordon RE, Iomini C. Primary cilia maintain corneal epithelial homeostasis by regulation of the Notch signaling pathway. Development. 2016;143:2160-71. https://doi.org/10.1242/dev.132704.

63. Blitzer AL, Panagis L, Gusella GL, Danias J, Mlodzik M, lomini C. Primary cilia dynamics instruct tissue patterning and repair of corneal endothelium. Proc Natl Acad Sci U S A. 2011;108:2819-24.

64. Bisgrove BW, Yost HJ. The roles of cilia in developmental disorders and disease. Development. 2006;133:4131-43.

65. Field D, Tiwari B, Booth T, Houten S, Swan D, Bertrand N, et al. Open software for biologists: from famine to feast. Nat Biotechnol. 2006;24:801-3. https://doi.org/10.1038/nbt0706-801.

66. Schindelin J, Arganda-Carreras I, Frise E, Kaynig V, Longair M, Pietzsch T, et al. Fiji: an open-source platform for biological-image analysis. Nat Methods. 2012;9:676-82. https://doi.org/10.1038/nmeth.2019.

\section{Publisher's Note}

Springer Nature remains neutral with regard to jurisdictional claims in published maps and institutional affiliations.

Ready to submit your research? Choose BMC and benefit from:

- fast, convenient online submission

- thorough peer review by experienced researchers in your field

- rapid publication on acceptance

- support for research data, including large and complex data types

- gold Open Access which fosters wider collaboration and increased citations

- maximum visibility for your research: over $100 \mathrm{M}$ website views per year

At BMC, research is always in progress.

Learn more biomedcentral.com/submissions 